\title{
Brustaugmentation mit autologem Fett - Erfahrungen aus 96 Operationen mit der BEAULI ${ }^{\mathrm{TM}}$-Methode
}

\author{
Breast Augmentation with Autologous Fat - Experience of 96 Procedures \\ with the BEAULI ${ }^{\mathrm{TM}}$-Technique
}

\author{
Autor \\ D. P. Münch \\ Institut \\ Chirurgische Praxis, Liposuction.ch, Wiedlisbach/Bern, Schweiz
}

\section{Schlüsselwörter \\ - Brustaugmentation \\ - autologer Fetttransfer \\ - BEAULI \\ - Liposuction}

Key words

- breast augmentation

- autologous fat graft

- BEAULI

- liposuction

eingereicht 14.8.2012 akzeptiert 13.3.2013

\section{Bibliografie}

Dol http://dx.doi.org/

10.1055/s-0033-1343396

Handchir Mikrochir Plast Chir

2013; 45: 80-92

(c) Georg Thieme Verlag KG

Stuttgart · New York

ISSN 0722-1819

Korrespondenzadresse

Dr. Daniel P. Münch

Chirurgische Praxis

Liposuction.ch

Wangenstraße 1

4537 Wiedlisbach

Schweiz

info@muench.ch

\section{Zusammenfassung}

In den letzten 30 Jahren hat das Interesse an einer Verwendung des körpereigenen Fettes zur ästhetischen Körperformung, speziell zur Augmentation der weiblichen Brust, kontinuierlich zugenommen. Fehlende Fremdkörperreaktionen, harmonisches Aussehen und ein natürliches Gefühl sind die Vorteile einer autologen FettzellTransplantation. Komplikationen wie Nekrosen, Infekte, Zystenbildungen, hohe Graftverluste sowie die Schwierigkeit, große Fettmengen in vernünftiger Zeit zu gewinnen boten in früheren Jahren Ansatz zu Kritik an der Methode des Eigenfett-Transfers. Mit der BEAULITM-Methode steht seit 2007 ein Verfahren zur rationellen Gewinnung und Aufbereitung größerer transplantabler Fettmengen zur Verfügung. Das Ziel dieser Arbeit ist es, die Operationstechnik präzise und reproduzierbar zu beschreiben sowie aufgrund eigener klinischer Erfahrungen einen umfassenden Überblick über den autologen Fetttransfer zu vermitteln. Zwischen dem 1. September 2010 und dem 30. Juni 2012 führte der Autor 96 Eigenfett-Transplantationen an 84 Patientinnen durch. Ausgewählt wurden Patientinnen zwischen 18 und 62 Jahren mit dem Wunsch nach einer moderaten Volumenvermehrung und Formung der Brüste. Die Fettgewinnung erfolgte mittels wasserstrahl-assistierter Liposuction (Bodyjet ${ }^{\mathrm{TM}}$ ) und anschließender Separierung der Fettzellen mit dem LipoCollector ${ }^{\mathrm{TM}}$. Postoperativ traten in keinem Fall Komplikationen auf. Die Resultate wurden im Rahmen einer Kontrolluntersuchung, eines Fotovergleichs und in der Beantwortung eines Fragebogens beurteilt. Bezüglich des Volumengewinns bzw. der Formverbesserung der Brüste waren $35 \%$ der Patientinnen mit dem Ergebnis sehr zufrieden, $42 \%$ zeigten sich zufrieden, $22 \%$ hätten sich noch mehr Volumen gewünscht, $1 \%$ war unzufrieden. Diese Studie zeigt, dass die autologe Fettzell-Transplantation

\section{Abstract}

Over the past 30 years, interest in the use of autologous fat for aesthetic body contouring, especially for breast augmentation has been continuously on the rise. The benefits of an autologous fat transplant include the absence of any inflammatory reaction to a foreign body, its harmonious appearance and a natural feeling. In earlier years, complications such as necrosis, infections or the formation of cysts, poor resorption rates as well as the difficulty of harvesting large amounts of fat within a reasonable amount of time provided grounds for criticism of the methodology of autologous fat transplantation. With the advent of the so-called BEAULI ${ }^{\mathrm{TM}}$ method, since 2007 a procedure is available for the efficient harvesting and processing of larger quantities of transplantable fat. The aim of the study is to describe the technique in detail and reproducibly and to present a detailed overview of autologous fat transfer due to the basis of our own clinical experience. Between 1 September 2010 and 30 June 2012 the author performed 96 fat transfer procedures on 84 patients. Patients aged 18-60 with a desire for a moderate augmentation of volume and shape of the breasts were selected for the procedure. The fat was harvested using water jet-assisted liposuction (Bodyjet $^{\mathrm{TM}}$ ) to flush out the fat cells and subsequent separation of the fat components with the LipoCollector $^{\mathrm{TM}}$. There were no occurrences of postoperative complications in any of the cases. The results were evaluated in the context of a checkup, a photographic comparison and with the completion of a questionnaire. With regard to the increase in size and/or shape enhancement of the breasts, $31 \%$ of the patients were very happy with the results, $45 \%$ indicated they were satisfied, $23 \%$ would have liked more volume, while $1 \%$ were dissatisfied. This study indicates that the autologous fat transplant into the female 
in die weibliche Brust mit der BEAULI ${ }^{\mathrm{TM}}$-Methode eine gute und sichere Methode ist, bei ausgewählten Patientinnen eine moderate und harmonische wirkende Brustvolumenvergrößerung sowie eine nachhaltige und natürliche wirkende Konturverbesserung zu erzielen. Weitere Studien mit hohen Fallzahlen und Beobachtungszeiten über mehrere Jahre sowie Guidelines der Fachgesellschaften könnten dazu beitragen, die Methode des Eigenfett-Transfers bezüglich Einheilungsrate, Effizienz und Sicherheit zu perfektionieren.

\section{Einleitung}

Autologer Fetttransfer zur Brustvolumenvergrößerung als Alternative zu Implantaten gewinnt zunehmend an Bedeutung. Unterschiedliche Langzeitergebnisse und offene Fragen bezüglich der Karzinogenese limitierten jedoch diesen Anwendungszweck. 1987 lehnte die American Society of Plastic Surgeons (ASPS) einen Fetttransfer zum Brustaufbau wegen der möglichen Karzinomentwicklung, der eingeschränkten Vorsorgeuntersuchung und des Auftretens von Verkalkungen ab. Technische Fortschritte in der Fettgewinnung und differenziertere Methoden zur Brustdiagnostik bildeten den Anlass, die Methode der Fettverpflanzung weiter $\mathrm{zu}$ entwickeln. 2009 revidierte die ASPS ihre Haltung und kam neu zum Schluss, dass der autologe Fetttransfer zum Brustaufbau bei technisch korrekter Durchführung durch einen erfahrenen Chirurgen und unter Beachtung der Risikofaktoren (familiäre Belastung Brustkrebs, Nachsorge) eine mögliche Therapieform darstellt. Illouz [1] berichtete 2009 über 25 Jahre Erfahrung in autologem Fetttransfer bei $820 \mathrm{~Pa}-$ tientinnen. Coleman [2,3] entwickelte 1998 ein Verfahren zur schonenden Gewinnung und Verpflanzung von Fettzellen, 2007 veröffentlichte er die Ergebnisse von Verläufen über mehrere Jahre nach Brustvergrößerungen mit transplantiertem Eigenfett. $\mathrm{Zu}$ den Vorteilen des Brustaufbaus mit autologem Fett zählen das Fehlen von Fremdkörperreaktionen, Abstoßungen und Narben sowie das natürlich und harmonisch wirkende Ergebnis. Die zur Fettgewinnung notwendige Liposuction erlaubt eine bei den meisten Patientinnen erwünschte zusätzliche Korrektur der Körperproportionen, womit 2 Eingriffe in einer Sitzung durchgeführt werden können. Die Patientenselektion, die Kompetenz des Chirurgen, die Methode der Gewinnung und der Aufbereitung des Fettes und die Injektionstechnik sind Faktoren, welche die Sicherheit, das Komplikationsrisiko und die Überlebensrate der transferierten Fettpartikel beeinflussen. In der Literatur finden sich unterschiedliche Angaben betreffend Patientenauswahl, Anästhesie, „Transfer-Tuning“ mittels Stammzellen oder PRP (Platelet-Rich Plasma), Einheilungsraten, Dauerhaftigkeit des Ergebnisses, Komplikationen, Einschränkungen späterer Brustvorsorgeuntersuchungen und möglicher Tumorinduktion. Beim Eigenfettbrustaufbau geht es um große Volumina (300$600 \mathrm{ml}$ aufbereitetes Fett), dadurch stellen sich auch Fragen im Hinblick auf die Sicherheit, den Aufwand, die Kosten und die Effizienz der Methode sowie die Dauer der Operation. Zu Beginn unserer Erfahrungsperiode mit 5000 Liposuctionen seit 1993 war für die Patientinnen die Frage nach einer Fettverpflanzung in die Brust kaum je ein Thema. In den letzten Jahren hingegen äußerten zunehmend mehr Patientinnen den Wunsch nach einer gleichzeitigen Eigenfettbrustvergrößerung. 2010 entschieden wir uns zum Wechsel von der Liposculpture-Methode nach Fournier zur BEAULI ${ }^{\mathrm{TM}}$-Methode (Berlin Autologous Lipotransfer) [4], wo die Technik der Wasserstrahl-assistierten Liposuc- breast using the BEAULI ${ }^{\mathrm{TM}}$ method represents an excellent, safe method for the achievement of a moderate and harmonious breast size augmentation as well as sustainable and naturallooking contour improvements in selected patients. Additional studies with a larger number of cases and longer observation periods over several years as well as guidelines from the professional associations could contribute to the further perfection of the autologous fat transplant method in terms of resorption rate, efficiency and safety.

tion (WAL) zum Einsatz kommt (Bodyjet ${ }^{\mathrm{R}}$, Firma Human Med). Mittels einer doppellumigen Kanüle werden die Fettzellen durch einen Sprühstrahl aus dem Zellverband gelöst und zusammen mit der infiltrierten Lösung abgesaugt. Das fortlaufende Spülen erlaubt das effiziente Arbeiten mit einem zellschonenden reduzierten Vakuum sowie die Verwendung nur 0,9 mm großen Kanülenöffnungen. Kleine Kanülenöffnungen sind notwendig, damit das Fett unter Anwendung von nur minimalem Druck die dünnen Filling-Kanülen passieren kann. Der Gehalt an vitalen Zellen ist vergleichbar zur Coleman-Methode [4]. Das abgesaugte Fett wird in einem Container gefiltert und gesammelt. Nach der Separierung von Wasser, allfälligen Blutbeimengungen und Öl durch einen Mikrofilter sind die Fettzellen bereit für das Umfüllen in die Injektionsspritzen. Eine für die Zellen potentiell schädigende Zentrifugation [5-8] ist nicht nötig. Durch die Technik der WAL wird das Fett in Form von sehr kleinen Partikeln zellschonend abgesogen. Weil bei kleineren Fettzellverbänden die Diffusionsstrecke minimal ist, wird die Einheilungsrate durch die erhöhte Vaskularisierung verbessert [9]. Mit dieser Methode lassen sich größere Fettmengen von hochwertiger Qualität in vernünftigem Zeitrahmen gewinnen.

\section{Patienten, Material und Methoden}

$\nabla$

Zwischen 1. September 2010 und 30. Juni 2012 hat der Autor bei 84 Patientinnen eine Liposuction mit anschließender autologer Fettzell-Transplantation zur Brustaugmentation durchgeführt. 11 Patientinnen wurden 2-mal operiert, 1 Patientin 3-mal. Insgesamt wurden 96 Prozeduren durchgeführt. Die Patientinnen waren zwischen 19 und 62 Jahre alt (Mittelwert: 36,7 Jahre), der Body Mass Index lag zwischen 18,6 und 29,8 (Mittelwert: 22,7). Berücksichtigt wurden Patientinnen mit dem Wunsch nach einer moderaten und natürlich wirkenden Volumenzunahme der Brüste, idealerweise mit dem gleichzeitigen Wunsch nach einer Korrektur von störenden Fettpolstern ( $\bullet$ Tab. 1). 35\% der Patientinnen entschlossen sich ausschließlich wegen des Wunsches nach einer massvollen Brustvergrößerung zu einer Operation.

Tab. 1 Indikationen zur Brustaugmentation mit Eigenfett.

\begin{tabular}{|l|l|}
\hline $\begin{array}{l}\text { Brustimplantat } \\
\text { Wunsch nach großem Volumen- }\end{array}$ & $\begin{array}{l}\text { Eigenfett-Transfer } \\
\text { Wunsch nach moderatem, } \\
\text { harmonischem Volumengewinn } \\
\text { und einer Konturverbesserung; } \\
\text { „sanfte“ und natürlich wirkende } \\
\text { Brustformung }\end{array}$ \\
\hline Fremdkörper ist egal & Abneigung gegen Fremdmaterial \\
\hline Raucherin & Nichtraucherin \\
\hline BMl unter 18,5 & $\begin{array}{l}\text { Normalgewicht oder leichtes } \\
\text { Übergewicht }\end{array}$ \\
\hline Ablehnung einer Liposuction & gleichzeitiger Liposuctionswunsch \\
\hline
\end{tabular}


Bei $21 \%$ der Patientinnen stand die Liposuction im Vordergrund und $44 \%$ der Fälle wünschten sowohl eine Liposuction als auch einen Brustaufbau. Bei einer Patientin erfolgte ein Brustwiederaufbau nach Ablatio in 3 Sitzungen. Bei 42 Operationen wünschten die Patientinnen neben der Volumenvermehrung gleichzeitig eine Korrektur bei asymmetrischen Brüsten, bei 3 Eingriffen wurde bei stärkerer Volumenasymmetrie nur eine Brust behandelt.

Ausgeschlossen wurden Patientinnen mit Diabetes mellitus, kardiovaskulären Erkrankungen, Blutungsanomalien oder Neigung zu Infektionen. Ebenfalls nicht berücksichtigt wurden Raucherinnen (über $3 \mathrm{Zig} /$ die) bzw. solche, welche sich nicht zu einem mindestens 2-monatigen Rauchstopp vor der Operation entscheiden konnten. Nicht in Frage kamen Patientinnen mit dem primärem Wunsch nach einer starken Volumenzunahme der Brüste in einer Operation (über $1 / 2$ Cup) oder einem BMI von unter 18,5. Zurückhaltend wurde die Indikation bei Patientinnen mit sehr schlaffen Brüsten nach starkem Gewichtsverlust oder mit dem ausdrücklichen Wunsch nach strafferen Brüsten gestellt.

Präoperativ wurden die Patientinnen über das Risiko der Bildung von Verkalkungen sowie über den zu erwartenden Volumengewinn im Sinne von festeren, praller wirkenden Brüsten (zirka 100-120 ml Volumenzuwachs oder zirka $1 / 2$ BH-Größe) informiert. Obligatorisch war die präoperative Durchführung einer Mammografie und/oder einer Sonografie als Ausgangsbefund (Patientin Nr. 1-56: nur wenn über 40 Jahre gemäß Empfehlung Ueberreiter [4]; Patientin Nr. 57-84: alle entsprechend Literaturrecherchen im Verlauf der Studie $[8,10,11])$. Die Patientinnen wurden auch dahin aufgeklärt, dass in seltenen Fällen, wo eine Verkalkung bezüglich Dignität nicht eindeutig beurteilt werden kann, eine Biopsie erforderlich sein könnte. Vor dem Eingriff wurden sowohl die abzusaugenden Zonen als auch die Filling-Zonen an den Brüsten im Stehen markiert. Fotos des Körpers von 3 Seiten sowie der Brüste von vorne und seitlich dokumentierten die präoperativen Befunde. Die Eingriffe erfolgten unter einer perioperativen Antibiose mit $2 \times 500$ mg Cefuroxim während 7 Tagen sowie einer prophylaktischen low-dose Heparinisierung (Clexane $\mathrm{R}^{\mathrm{R}} 1 \times 20 \mathrm{mg}$ s.c.) während 5 Tagen.

Tab. 2 Zusammensetzung der Infiltrations- und Spüllösung.

\begin{tabular}{lc}
\hline Lidocain $1 \%$ & $50 \mathrm{ml}$ \\
\hline Adrenalin $1 \mathrm{mg}$ & $1 \mathrm{ml}$ \\
\hline Natriumbicarbonat $8,4 \%$ & $12,5 \mathrm{ml}$ \\
$\mathrm{NaCl} 0,9 \%$ & $1000 \mathrm{ml}$
\end{tabular}

\section{Infiltration}

Alle Eingriffe erfolgten ambulant, in lokaler Betäubung und unter einer Prämedikation mit 7,5 mg Dormikum p/o. Je nach Bedarf erfolgte eine zusätzliche Sedation mit Enthonox ${ }^{\mathrm{R}}$, einem Gasgemisch aus 50\% Distickstoffmonoxid („Lachgas“) und 50\% Sauerstoff. Enthonox ${ }^{R}$ ist ein stark wirkendes Analgetikum mit einem schnellen Wirkungseintritt, es ist einfach zu verabreichen und wird nach Beendigung rasch aus dem Körper ausgeleitet. Der Patient bleibt dabei immer ansprechbar und kann sich selbstständig in die optimale Position drehen, was die Durchführung der Liposuction erleichtert.

Durch kleine Stichinzisionen wird die 2,5 mm Infiltrationskanüle in die subkutane Fettschicht der zuvor im Stehen markierten Behandlungszonen eingeführt. Die auf $37^{\circ}$ vorgewärmte Anästhesieflüssigkeit ( $\bullet$ Tab. 2) wird am Ende der Kanüle durch eine um $30^{\circ}$ von der Längsachse abgewinkelte Öffnung fächerförmig und dadurch gleichmäßig in die Fettschicht verteilt. Die Infiltration der Lösung erfolgt pulsierend mit einem Flow von $110 \mathrm{ml} /$ min und einem Druck von 50 bar, die Eindringtiefe der Flüssigkeit in das Fettgewebe beträgt so $40-50 \mathrm{~mm}$. Die Kanüle wird fächerförmig und langsam, dem pulsierenden Sprühstrahl folgend, hin und her bewegt und gleichzeitig um die Längsachse gedreht. Dies erlaubt eine möglichst schmerzfreie und schonende Infiltration der subkutanen Fettschicht, außerdem wird das Fettgewebe durch den Wasserstrahl sanft disseziert und auf die nachfolgende Liposuction vorbereitet. Bei sehr empfindlichen Patienten ist eine Präinfiltration von Vorteil. Dabei werden zunächst nur geringe Volumina der Lösung im Randbereich der Absaug-Zonen und an empfindlichen Arealen infiltriert. Das Gewebe wird nicht wie bei der typischen Tumeszenztechnik prall gefüllt (wodurch die Fettzellen geschädigt werden könnten), sondern das Ziel ist eine Basisinfiltration der subkutanen Fettgewebeschicht. Auch bei den Brüsten erfolgt die Infiltration zunächst ringförmig um die Brust herum („Ringblock“), danach werden die subkutanen und subglandulären Schichten infiltriert.

\section{Lipoaspiration}

Nach einer Einwirkzeit der Anästhesie von 20 min erfolgt die Absaugung mit einer doppellumigen Kanüle von $25 \mathrm{~cm}$ Länge und 3,8 mm Durchmesser mit scharfem Kantenschliff ( $\bullet$ Abb. 1). Die Infiltrationslösung bleibt in ihrer Zusammensetzung unverändert. Analog der vorgängigen Infiltrationsphase wird die Anästhesieflüssigkeit an der Spitze der Kanüle fächerförmig im Gewebe verteilt, dadurch wird der anästhetische Effekt auch in der Absaugphase kontinuierlich aufrechterhalten. Durch das fortlaufende Spülen wird außerdem die Belastung durch die in

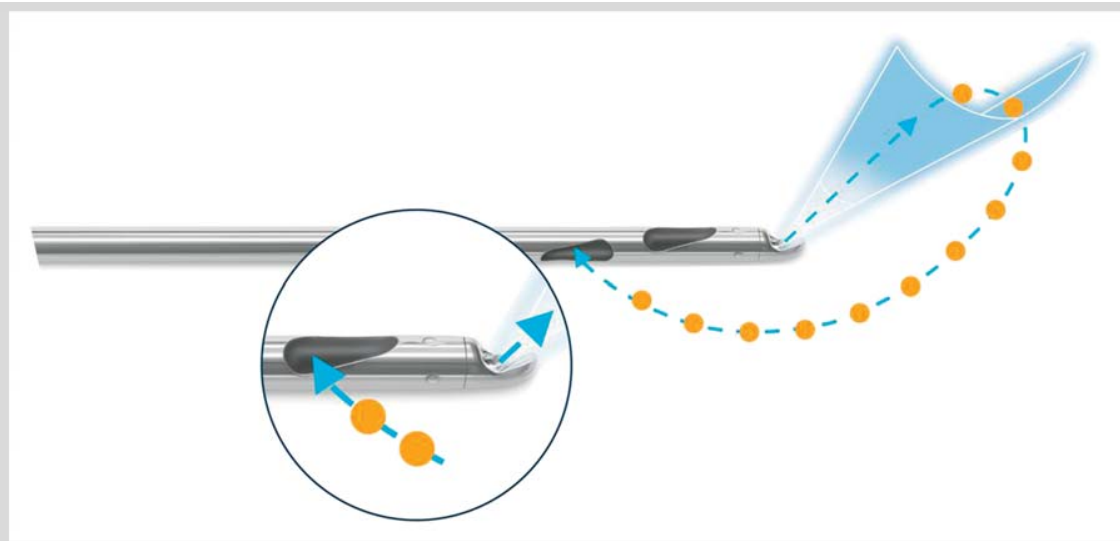

Abb. 1 Doppellumige Liposuctions-Kanüle (Fa. Human Med AG). 
der Lösung enthaltenden Medikamente sowohl für den Patienten als auch für die gewonnenen Fettzellen reduziert. 4 seitlichzirkulär angeordnete, längsovale Saugöffnungen im Abstand von 25 mm zur Kanülenspitze und mit einem wirksamen Durchmesser von $0,9 \mathrm{~mm}$ erlauben die Gewinnung von sehr kleinen Fettzellverbänden, sodass diese die $2 \mathrm{~mm}$ Fettfilling-Kanülen nicht verstopfen. Der Unterdruck wird durch das Verschließen eines Bypassloches mit dem Daumen am Handstück der Kanüle aufgebaut und kontrolliert. Um die Fettzellen möglichst schonend zu behandeln, wird der Druck auf - 0,5 bis maximal -0,55 bar begrenzt. Unter langsamen, gleichmäßigen Hin- und Herbewegungen werden die Adipozyten mittels des pulsierenden Wasserstrahls (Druck: 30 bar, Flow: $90 \mathrm{ml} / \mathrm{min}$ ) aus ihrer extrazellulären Matrix „herausgespült“ und schonend abgesogen. Wie bei jeder Liposuction üblich, werden die Zonen gleichmäßig fächerförmig (Criss-Cross Technik) behandelt. Am Ende dieses Infiltrations/Absaugevorganges werden die Inzisionsstellen offenbelassen, nach Austritt der restlichen Spülflüssigkeit verschließen sich diese spontan innert 24h. Bei 58 Operationen erfolgte die Liposuction am Bauch, bei 57 Eingriffen an den Hüften und in 71 Sitzungen wurde das Fett an den Oberschenkeln gewonnen.

\section{Fettaufbereitung}

Das Aspirat wird durch den zwischengeschalteten, geschlossenen und sterilen Lipocollector ${ }^{R}$ gesammelt. Ein Sieb mit 2,0 mm schmalen Schlitzen filtert gröbere Bindegewebsstrukturen oder größere Fettpartikel, welche die Filling-Kanüle verstopfen können und eine schlechte Einheilungsrate haben, aus dem Aspirat. Ein Feinfilter mit einem Porendurchmesser von $250 \mu \mathrm{m}$ trennt die gewonnenen Fettzellen von überschüssiger Flüssigkeit ( $\bullet$ Abb. 2). Ueberreiter bestimmte in seiner mit der gleichen Technik durchgeführten Serie einen Restanteil von 30\% Flüssigkeit [4]. Diese Verdünnung ist erwünscht, denn sie erlaubt eine schonende Injektion der Fettzellen in die Brust und unterstützt deren gleichmäßige Verteilung im Empfängergewebe. Nach Abschluss der Liposuction werden die Adipozyten durch eine Öffnung im Deckel des Collectors mit einer 1,9 mm-Kanüle unter sanftem Sog in $60 \mathrm{ml}$ Spritzen umgefüllt. Dieses Manöver erfolgt ohne Luftkontakt, was bezüglich Sterilität und minimaler Traumatisierung der Fettzellen wichtig ist. Anschließend wird das Aspirat mittels Luer-Luer Verbindungsstück in $10 \mathrm{ml}$ Spritzen umgefüllt. Die so aufbereiteten Fettpartikel sind dann zur Reinjektion bereit. Die $10 \mathrm{ml}$ Spritzen werden nun separat für die rechte und für die linke Brust abgezählt, falls eine Asymmetrie

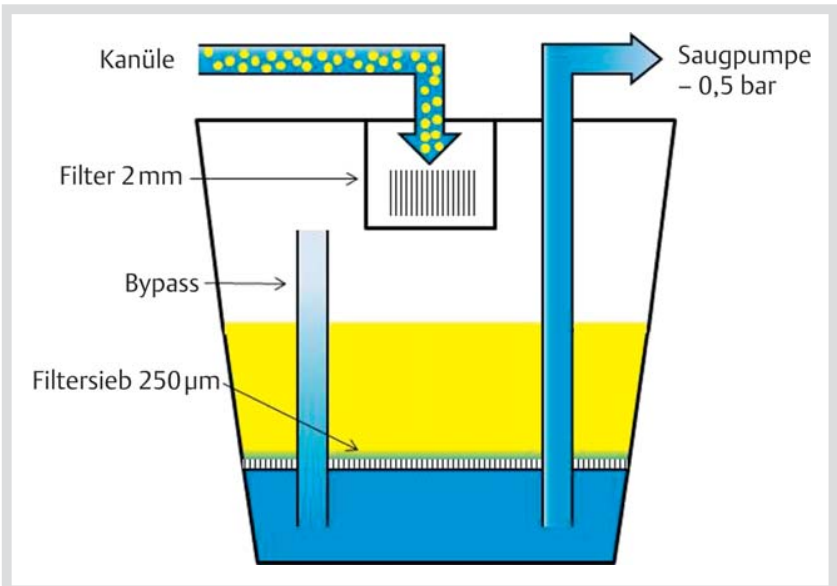

Abb. 2 Funktionsschema Lipo Collector ${ }^{\mathrm{TM}}$ (Fa. Human Med AG). der Brüste ausgeglichen werden muss, kann dies entsprechend berücksichtigt werden.

\section{Reinjektion}

Die Reinjektion der Fettzellen erfolgt von 2 kleinen Stichinzisionen laterocaudal und parasternal, mit jeweils $2 \mathrm{~cm}$ Abstand zur Brust ( $\bullet$ Abb. 3). Zur Anwendung gelangt eine $10 \mathrm{ml}$ Spritze mit einer stumpfen 2,7 mm Kanüle (12G) von $150 \mathrm{~mm}$ Länge mit 2 seitlichen Öffnungen am Kanülenende. Die Infiltration erfolgt ausschließlich in der subkutanen und subglandulären Fettschicht sowie im Bereich der Pectoralismuskulatur, keinesfalls jedoch intraglandulär. Etwa $2 / 3$ werden subkutan und $1 / 3$ intrapectoral verteilt. In den Pectoralismuskel kann in einer Operation zirka $50 \mathrm{ml}$ Volumen infiltriert werden, hier wird die Kanüle unmittelbar oberhalb der Rippen geführt. Insbesondere bei eher straffen Brüsten werden der Oberkörper der Patientin $45^{\circ}$ aufgerichtet und die Arme seitlich am Körper gelagert. Dadurch ist das Gewebe weniger gespannt, die zu infiltrierenden Schichten werden breiter und die Kanüle lässt sich leichter und präziser führen. Die Infiltration erfolgt durch sanften, gleichmäßigen Druck auf den Spritzenstempel unter gleichzeitigem Zurückziehen der Kanüle. Die Kanüle wird dabei gleichzeitig in der Längsachse fortlaufend nach links und rechts gedreht. Dadurch werden die Adipozyten linear-perlschnurartig und in feinen Portionen verteilt. Eine Infiltration nur beim Zurückziehen verhindert auch eine versehentliche Injektion in Gefäße. Von beiden Stichinzisionen aus wird die ganze Brust gleichmäßig, fächerförmig und in allen Schichten infiltriert (3D-Filling). Wichtig für das spätere Ergebnis ist auch ein Filling in Richtung Dekolleté. Das Ziel ist eine Bienenwabenstruktur von Mikrotunnels von maximal 2-3 mm Dicke. Diese Vorgehensweise ist die Voraussetzung, damit die Diffusionsstrecke minimal ist und möglichst viele Fettzellen Anschluss an ein Blutgefäß finden. Damit lässt sich eine möglichst hohe Einheilungsrate erzielen und es wird vermieden, dass Adipozyten absterben und sich nachfolgend Ölzysten und Verkalkungen bilden. Um die Fettzellen keinem zusätzlichen Druck auszusetzen, wird darauf geachtet, dass das Gewebe nicht „überfüllt“ wird. Die Brüste sollten „fest, aber nicht gespannt sein“ [4], das heißt, mit der Fingerkuppe noch eindellbar sein. Bei kleinen, straffen Brüsten liegt das Volumen bei 100$120 \mathrm{ml}$, bei größeren, eher schlaffen Brüsten bei $250-280 \mathrm{ml}$. In unserer Serie lag der Mittelwert des infiltrierten Volumens bei $170 \mathrm{ml}$ pro Seite (Minimum: $70 \mathrm{ml}$, Maximum $250 \mathrm{ml}$ ). Bei asymmetrischen Brüsten wurde ein Volumenausgleich angestrebt, indem die kleinere Brust maximal gefüllt wurde und bei

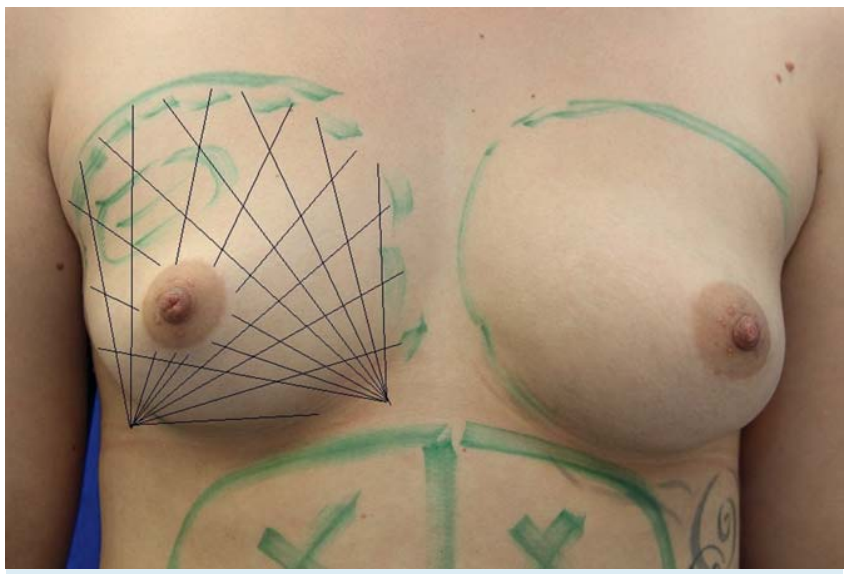

Abb. 3 Fächerförmige Fett-Injektion. 
der größeren Brust anschließend entsprechend weniger Volumen transplantiert wurde. Im Durchschnitt betrug die Operationszeit in unserer Serie $2 \mathrm{~h}$ und $15 \mathrm{~min}$.

\section{Nachbehandlung}

Die Infiltrationsinzisionen werden mit Pflastern versorgt, die Inzisionen der Liposuction werden offenbelassen. Nach der Operation legen wir einen Baumwollverband locker um die Brüste, eine Warmluftdecke (Bair Hugger ${ }^{R}$ ) hält die Temperatur im Brustbereich konstant bei $37^{\circ}$ und regt so die Durchblutung an. Die Absaugzonen werden flächenhaft auf $16^{\circ}-18^{\circ}\left(\right.$ Hilotherm $\left.^{\mathrm{R}}\right)$ gekühlt, dies reduziert die Schwellneigung, beugt Hämatomen vor und wirkt schmerzlindernd. Um eine möglichst hohe Einheilungsrate zu erreichen werden die Patientinnen angehalten, die Brüste während 2-3 Tagen vor Druck und lokaler Auskühlung zu schützen. Druck auf die Brüste sollte dringend vermieden werden, da die fragilen transplantierten Zellen geschädigt werden können oder die Durchblutung eingeschränkt werden kann. Schutz vor lokaler Auskühlung fördert die Durchblutung und verbessert die Einheilungsrate. Ab dem dritten postoperativen Tage empfehlen wir das Tragen eines leichten Sport-BHs ohne Bügel.

\section{Komplikationen}

Nach keiner der 96 Operationen traten postoperative Komplikationen wie Infekte, Nekrosen, Blutungen, Serome oder thromboembolische Ereignisse auf. Neben den üblichen Schwellungen und Druckdolenzen waren nur selten vorübergehende Rötungen oder Hautirritationen zu verzeichnen. In 5 Fällen (4,8\%) fanden sich postoperativ subkutane Hämatome, in 2 Fällen $(1,9 \%)$ Konturunregelmäßigkeiten und in 2 Fällen (1,9\%) Asymmetrien. Alle diese genannten Befunde bildeten sich aber innerhalb von maximal 3 Wochen vollständig zurück. Während des Eingriffes und in den ersten $24 \mathrm{~h}$ danach traten auch in keinem Fall hämodynamische Instabilitäten, Nausea, Parästhesien oder ähnliche Komplikationen auf. 48\% der Patientinnen bezeichneten die Schmerzen in der postoperativen Phase mit wenig, 39\% mit erträglich und 13\% mit eher stark. Bei 53\% der Fälle betrug die Arbeitsunfähigkeit (Bürotätigkeit) nach dem Eingriff bis zu 2 Tage, bei $40 \%$ lag sie zwischen 3 und 4 Tagen und bei $7 \%$ zwischen 5 und 7 Tagen.

\section{Ergebnisse}

$\nabla$

Die Erfolgskontrolle basiert auf dem Vorher-Nachher Vergleich von seitliche aufgenommenen Fotografien (Mamille li - Mamille re - Objektiv auf einer Achse liegend), den subjektiven Aussagen der Patientinnen bei der Nachuntersuchung und der Auswertung eines Fragebogens. Mittels volumetrischer MRTAuswertungen könnte der Volumenzuwachs exakt belegt werden, diese Methode würde aber den Aufwand dieser „one surgeon study“ finanziell und personell übersteigen. Die durchschnittliche Beobachtungszeit lag bei 4,7 Monaten (0,5-18 Monate). Bezüglich der Formverbesserung der Brüste waren 35\% der Patientinnen mit dem Ergebnis sehr zufrieden, $42 \%$ zeigten sich zufrieden, $22 \%$ hätten sich noch mehr Volumen gewünscht und $1 \%$ war unzufrieden ( $\bullet$ Abb. 4). Subjektiv bezeichneten $35 \%$ der Patientinnen das Ergebnis mit dem Gefühl von volleren und pralleren Brüsten, 31\% als optischen Unterschied, 9\% als mit mehr Brustumfang und 25\% als nur minimes Volumenplus ( $\bullet$ Abb. 5). In diese letztgenannte Kategorie fallen vorwiegend ältere Patientinnen, solche, wo nur wenig Eigenfett gewonnen werden konnte oder Patientinnen mit zwischenzeitlichen Gewichtsabnahmen. Objektiv fand sich in jedem Fall der nachkontrollierten Patientinnen fotografisch ein Unterschied vorher/ nachher. Fallbeispiele mit prä- und postoperativen Fotos dokumentieren Resultate aus unserer Serie ( 0 Abb. 6-15). 49\% der Patientinnen würden sich gerne einer weiteren Transfer-Operation unterziehen lassen, $24 \%$ sind unsicher und 27\% wünschten keinen zusätzlichen Eingriff.

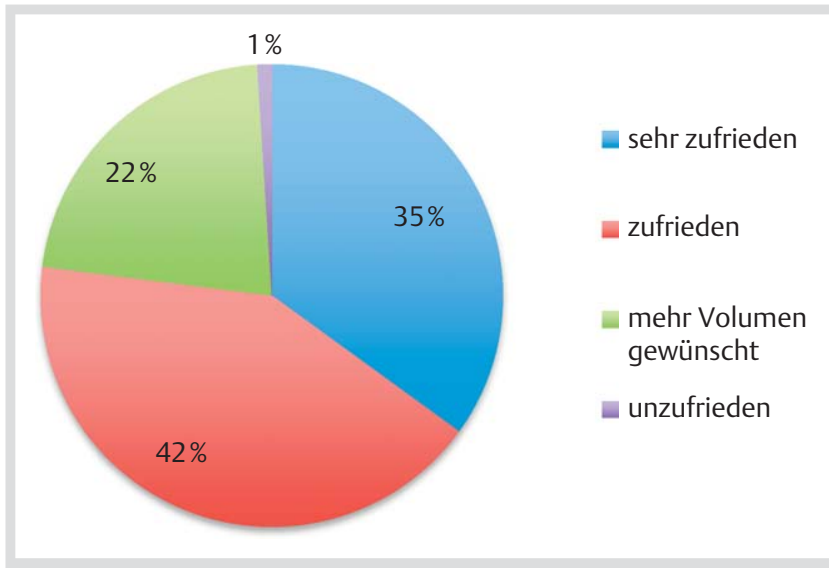

Abb. 4 Subjektive Beurteilung des Resultats.

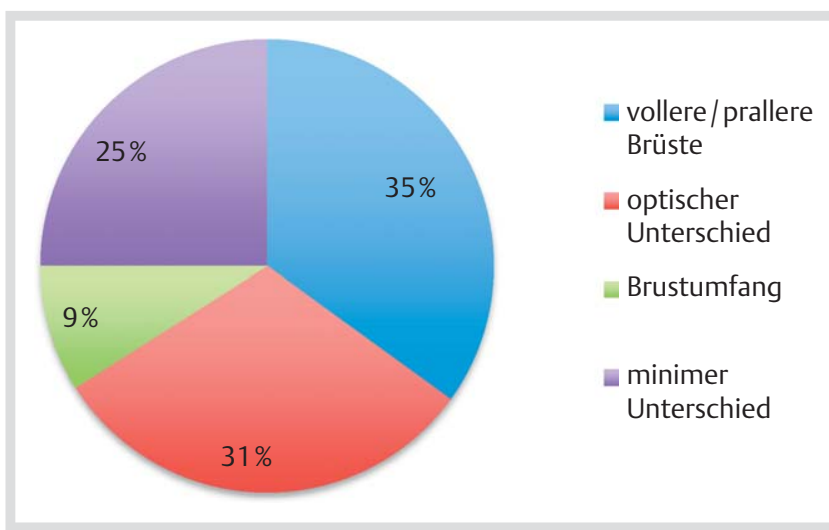

Abb. 5 Subjektive Beurteilung der Brustvolumenänderung.

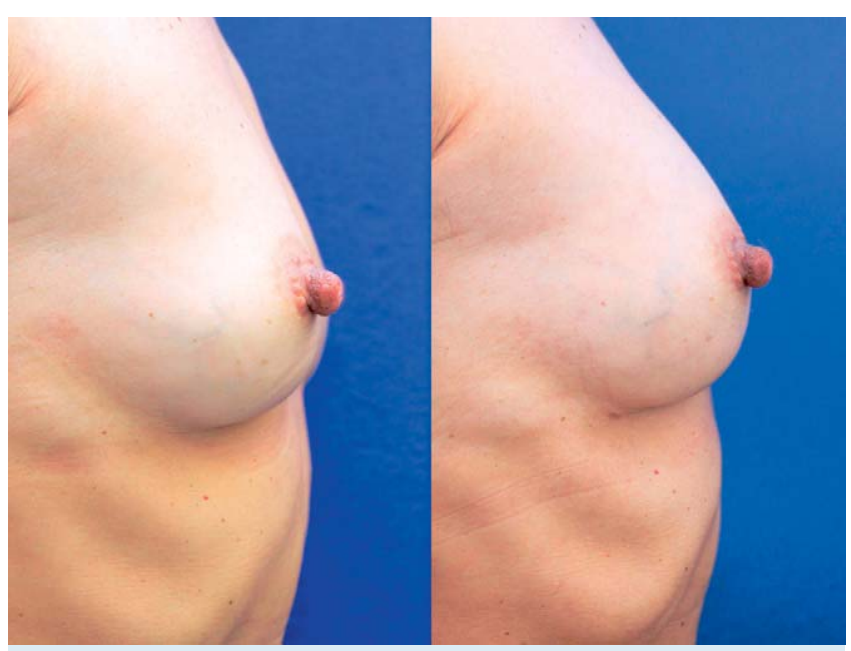

Abb. 6 52-jährige Patientin, 3 Monate nach Brustfilling. 


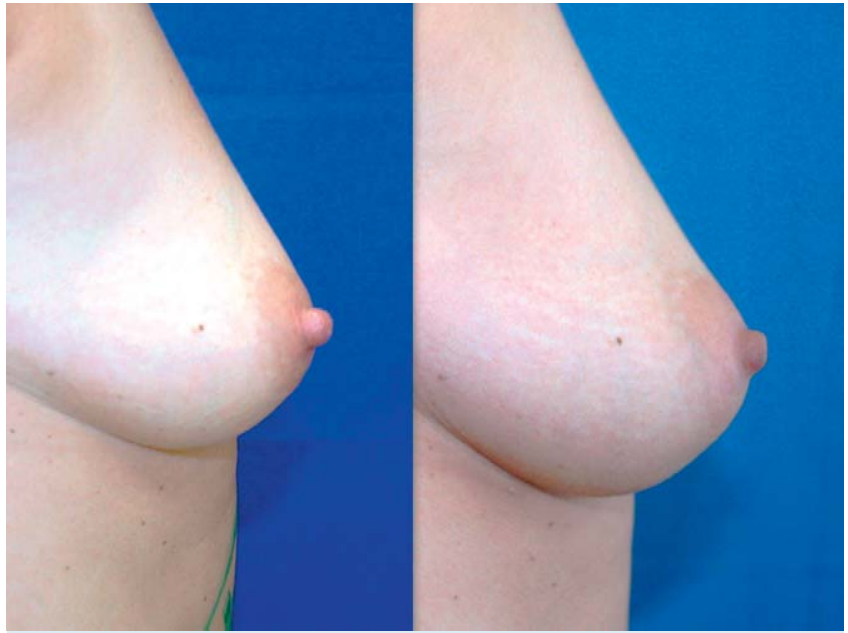

Abb. 7 44-jährige Patientin, 7 Monate nach Brustfilling.

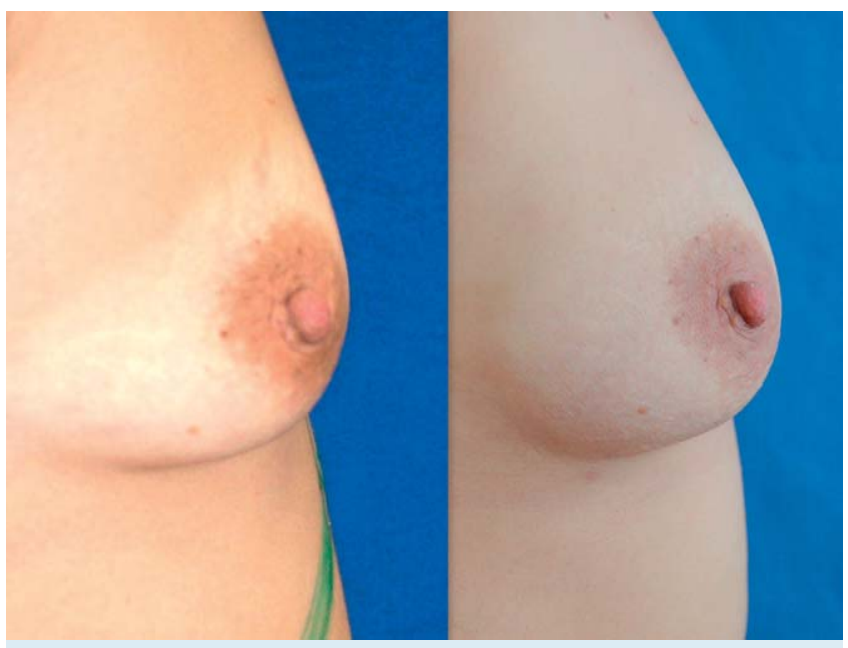

Abb. 8 31-jährige Patientin, 4 Monate nach Brustfilling.

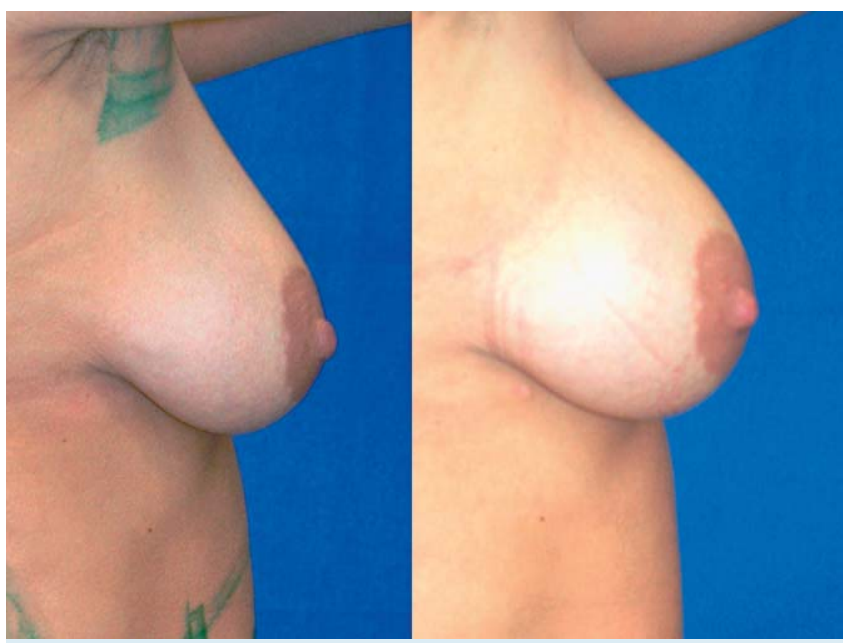

Abb. 9 46-jährige Patientin, 5 Monate nach Brustfilling.

\section{Diskussion}

$\nabla$

Aus der Liposuction zur Körperformung hat sich die Methode der autologen Fettzell-Transplantation zur Korrektur von Körperdefekten oder zu kosmetischen Indikationen entwickelt. Zahlreiche Varianten der Gewinnung, der Aufbereitung und des

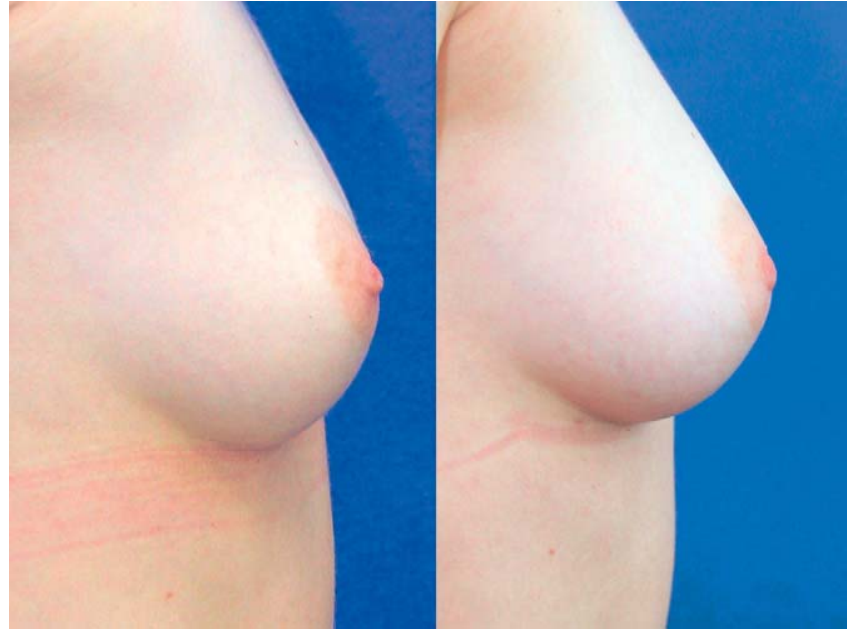

Abb. 10 27-jährige Patientin, 2 Monate nach Brustfilling.

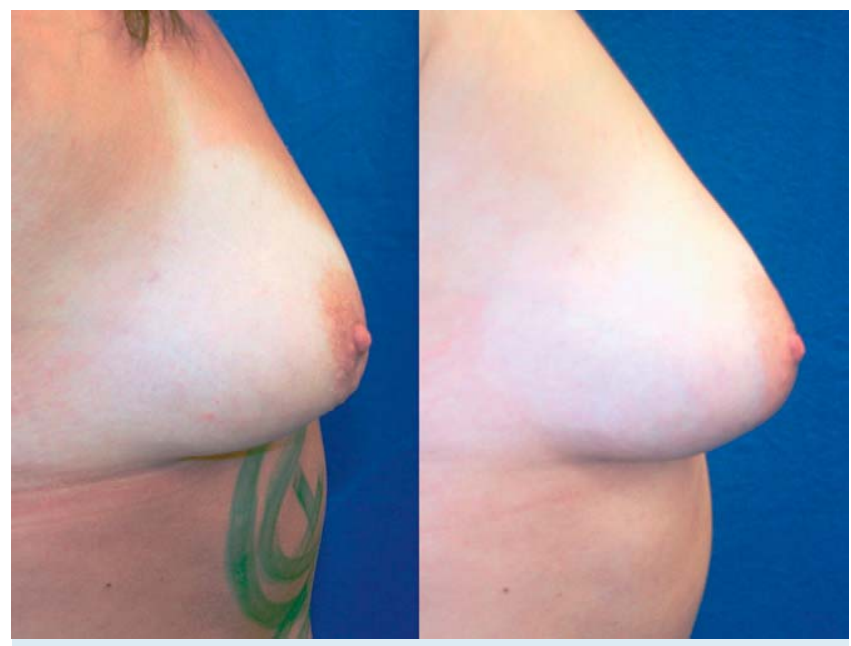

Abb. 11 30-jährige Patientin, 5 Monate nach Brustfilling.

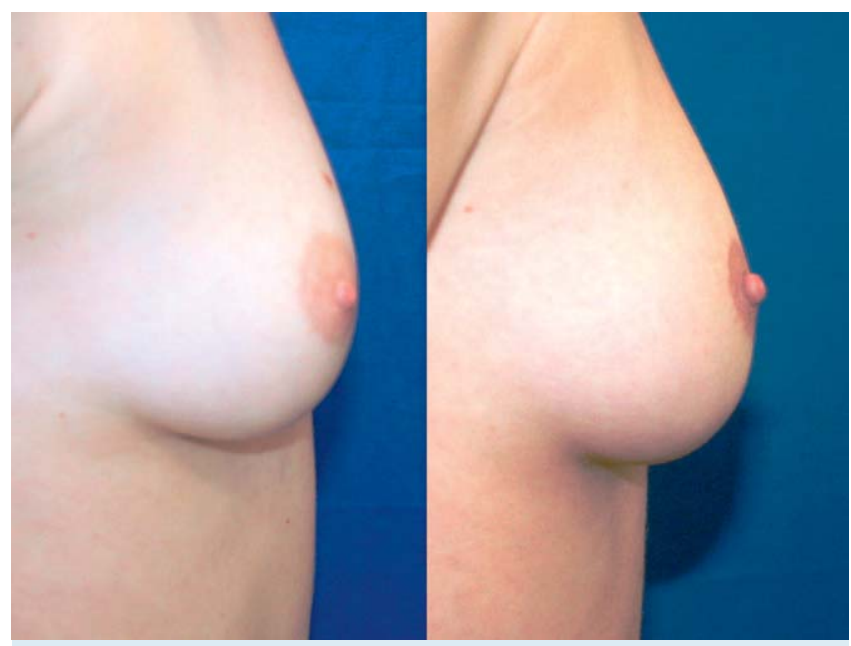

Abb. 12 31-jährige Patientin, 6 Monate nach Brustfilling.

Transfers werden beschrieben. Auch bezüglich Effektivität und Einwachsraten sind die Diskussionen im Gang. Die Geschichte der Fettverpflanzung begann 1893 mit der Verpflanzung von Fettstückchen in das Gesicht eines Tuberkulose-Patienten. Fournier [12] propagierte als Erster, Fett mittels Spritze und Nadel zu gewinnen und konnte nachweisen, dass mittels Spritzenaspira- 


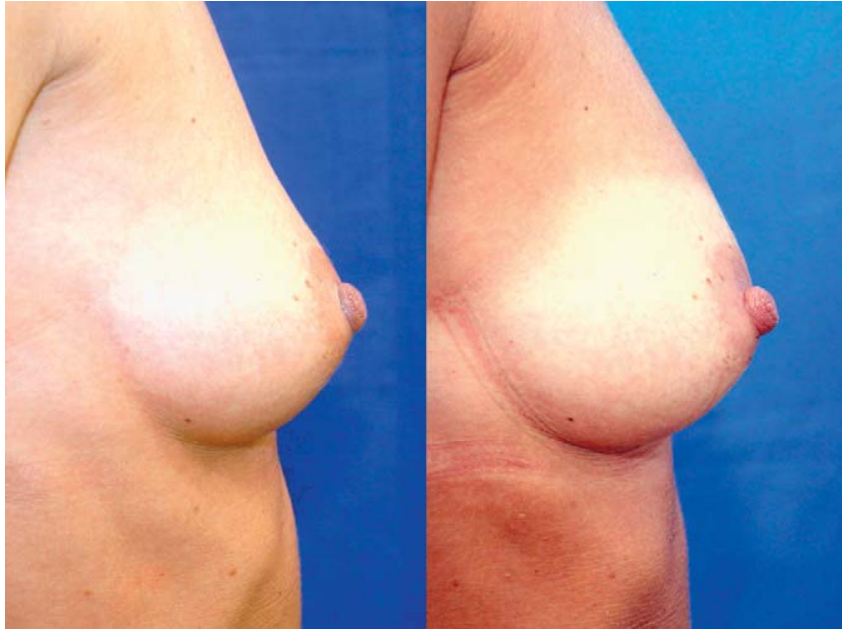

Abb. 13 40-jährige Patientin, 5 Monate nach dem 2. Brustfilling.

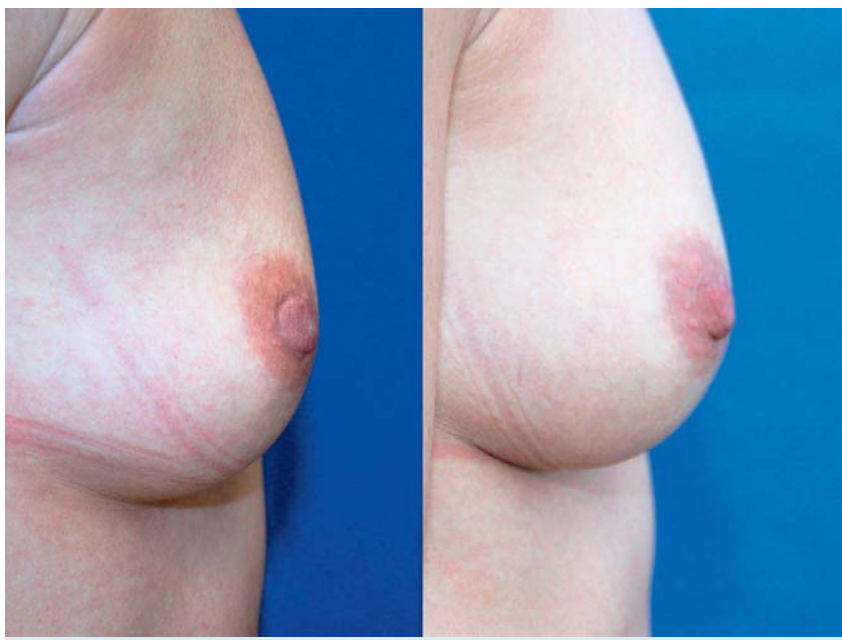

Abb. 14 39-jährige Patientin, 6 Monate nach dem 2. Brustfilling.

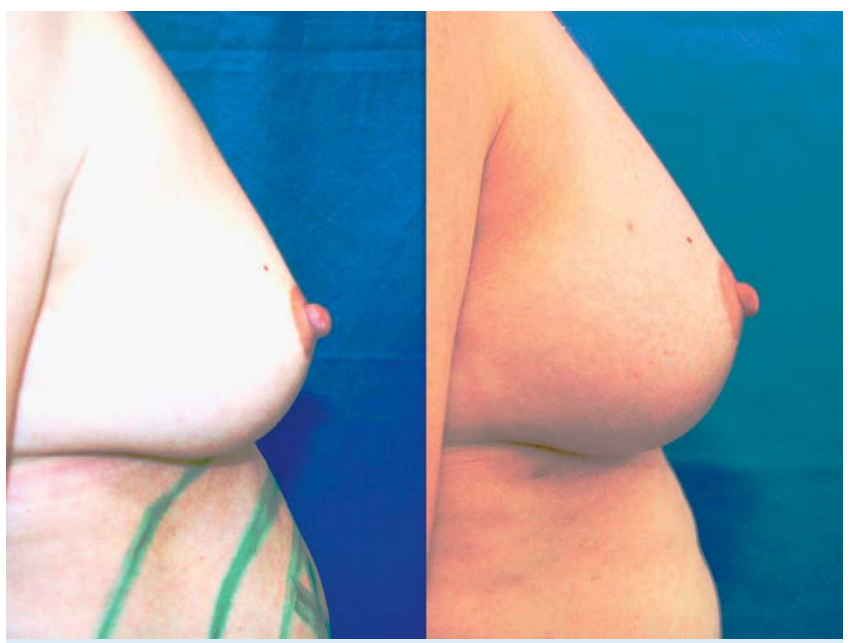

Abb. 15 41-jährige Patientin, 6 Monate nach dem 2. Brustfilling.

tion intakte Adipozyten gewonnen werden können. Coleman [3] bewies 1998 in seinen Arbeiten über die Lipostructure-Methode die grundsätzliche Möglichkeit, autologe Fettzellen erfolgreich zu verpflanzen. Seine Methode bei größeren Transplantationsvolumen ist allerdings sehr zeitaufwändig: mit $5 \mathrm{ml}$ Spritzen wird das Fett manuell abgesaugt, zentrifugiert, in $2 \mathrm{ml}$ Spritzen umgefüllt und anschließend in die Brust injiziert. Eine Brustvergrößerung dauert so bis zu 8 Stunden, was bezüglich Aufwand, Kosten und Belastung des Patienten unvorteilhaft ist.

Hauptziel eines Eigenfett-Transfers ist es, einen maximalen, nachhaltigen Volumengewinn im Empfängergebiet zu erreichen. Erwiesen ist, dass Fettzellen keine exzessiven Manipulationen wie Unterkühlen, Einfrieren, mechanische Beanspruchung oder starke Druckschwankungen tolerieren. Eine autologe Eigenfettzell-Transplantation erfordert deshalb ein delikates Handling, es muss die richtige Technik zum Einsatz kommen und auch den Details muss die nötige Beachtung geschenkt werden. Traumatische Fettgewinnung, falsche Kanülen, zu viel negativer Druck, Blutbeimengungen, Luftexposition oder Kontaminationen sind mögliche Ursachen für einen Misserfolg. Alter des Patienten, genetische Disposition, Raucheranamnese oder Ernährungsgewohnheiten sind ebenso Faktoren, die Einfluss auf das Ergebnis haben können [1,13].

Nicht aufbereitetes Fett enthält 3 Hauptkomponenten: intakte Fettzellen, verflüssigtes Fett und serosanguinöse Flüssigkeit. Diese Anteile müssen separiert werden, um eine optimale Konzentration an intakten Adipozyten ohne Blut- und Ölbeimengungen zu gewinnen. Voraussetzung für das Überleben von Fettzellen ist ein möglichst großes Verhältnis von Oberfläche zu Volumen. Kleine, tropfenförmige Transplantate weisen gegenüber größeren Fettgewebestückchen eine relativ große Oberfläche auf. Dies beschleunigt die Vaskularisation und führt $\mathrm{zu}$ einer besseren Resorptionsrate. Um eine hohe Überlebensrate von großen Transplantat-Volumina zu erreichen, müssen genügend kleine Fettgewebspakete in großer Zahl, absolut gleichmäßig und fein im gut durchbluteten Empfängergewebe verteilt werden. Je größer das Transplantat, umso geringer sind die Chancen, dass es einwächst. Das Überleben von transferierten Fettzellen vor Anschluss an ein Blutgefäß beruht auf Diffusion, wobei die Diffusionsstrecke bei maximal $100 \mu \mathrm{m}$ liegt [9]. Fettzellen weisen einen Durchmesser von $50-150 \mu \mathrm{m}$ auf, $1 \mathrm{~mm}^{3}$ enthält zirka 1000 Adipozyten. Die Mehrzahl der überlebenden transferierten Fettzellen liegt im Abstand von maximal $1 \mathrm{~mm}$ zu den Empfängerzellen. Bei Fettgewebsstückchen von bis maximal $1 \mathrm{~mm}$ Durchmesser kann somit davon ausgegangen werden, dass sie einwachsen. Bis zu Beginn der Angiogenesis dauert es zwischen 24-48 h [3] und 4 Tagen [14]. Daher ist es wichtig, dass während dieser Zeitspanne kein Druck auf das Empfängergebiet ausgeübt wird und die Körpertemperatur nicht unterschritten wird. Adipozyten sind sehr fragil, die extrakorporelle Überlebenszeit beträgt bei moderaten Temperaturen nur 4-6h [15].

\section{Anästhesie und Thromboseprophylaxe}

Der Eigenfett-Transfer wird von den meisten Autoren in Lokalanästhesie durchgeführt. Die Frage, ob aufgrund des potentiell schädigenden Einflusses von Lidocain auf die Zellvitalität eine Allgemeinnarkose bevorzugt werden sollte, wird nicht eindeutig beantwortet [16,17]. Shiffman [14] empfiehlt eine Allgemeinnarkose, weil die Klein-Lösung unphysiologisch ist und die Fettzellen aufgrund des onkotischen Gefälles Flüssigkeit aufnehmen. Die Frühmobilisation, das Tragen der Kompressionskleidung, der hämodilutive Effekt der Klein-Lösung und die thrombozytenaggregationshemmende Wirkung von Lidocain sind Faktoren, welche die Thromboseprophylaxe unterstützen. In der Literatur werden Einzelfälle von Lungenembolien beschrieben [5]. Beim Fettzelltransfer in die Brust scheint eine Thromboseprophylaxe mit niederdosiertem Heparin (20 mg s.c./die) indiziert zu sein. 
Offen bleibt die Frage, ob durch eine Heparinisierung auch die Resorptionsrate verbessert werden kann.

\section{Donorzone}

Bei eher hellhäutigen, tendenziell übergewichtigen und unsportlichen Patientinnen mit weichen und prominenten Fettpolstern lässt sich das Fett rasch, unproblematisch, in genügenden Mengen und guter Qualität gewinnen. Bei jüngeren, gut trainierten, eher dunkelhäutigen Patientinnen mit straffem und derbem Gewebe erweist sich die Fettgewinnung oft als eine etwas mühsame Prozedur. Bezüglich der optimalen Donorzone werden unterschiedliche Ansichten vertreten. In erster Linie richtet man sich nach den Wünschen der Patientin. Bei leicht untergewichtigen Patientinnen ist man ohnehin auf alle verfügbaren Zonen angewiesen, hier können keine Prioritäten gesetzt werden. Bei schlanken Patientinnen bietet sich ein großflächige aber nur sehr dünnschichtige Liposuction im Bereich des Gesäßes und an der Oberschenkel-Vorderseite als zusätzliche Zone an. Hier lässt sich unter Beibehaltung der Form und mit kaum bemerkbarem Volumenrückgang kompaktes Fett gewinnen. Als bevorzugte Spenderzonen werden die diätresistenten oder genetischen Fettdepots (mit $\alpha-2$-Rezeptoren) genannt [14]. Diese Zonen bieten sich in erster Linie an, da sie meistens Wunschzonen der Patientin sind und genügend Fett in guter Qualität gewonnen werden kann. Weniger geeignete Zonen sind Oberbauch und Flankenbereich, da hier das Fett oft von fibrösen Strängen durchzogen ist. Bevorzugt werden sollten auch Zonen mit relativ schlechter Durchblutung [18], Fettzellen von diesen Regionen weisen eine reduzierte metabolische Aktivität auf, sind resistenter und weisen eine verbesserte Überlebensrate auf.

Rohrich [19] verglich mittels Liposuction gewonnenes Fett aus unterschiedlichen Spenderzonen und fand keine Unterschiede bezüglich Viabilität der Fettzellen. Postuliert wird, dass im Bereich der oberflächlichen Fettschichten offenbar am meisten Stammzellen vorkommen. Stammt das Transplantat aus „primären Fettdepots“, also aus diätresistenten oder genetischen Zonen, kann davon ausgegangen werden, dass dieses Charakteristikum auch im Empfängergebiet erhalten bleibt. Fettzellen mit $\alpha$-2-Rezeptoren sind antilipolytisch und reagieren schlecht auf Diät. Diese Adipozyten reagieren weniger auf spätere Gewichtsschwankungen und scheinen eine bessere Überlebensrate als Adipozyten mit $\beta$-1-Rezeptoren aufzuweisen [18]. Untersuchungen zeigten, dass bei Patientinnen mit späterer Gewichtszunahme nach einem Eigenfetttransfer aus diätresistenten Zonen das Brustvolumen überproportional zunahm während an den abgesaugten Stellen nur minimale Veränderungen zu beobachten waren [17]. Umgekehrt kann davon ausgegangen werden, dass sich in solchen Fällen bei späterer Gewichtsreduktion das Brustvolumen auch nicht proportional zurückbildet.

\section{Tumeszenzlösung}

Die Tumeszenzlösung setzt sich in der Literatur weitgehend aus der gleichen Formel zusammen ( $\bullet$ Tab. 2). In jüngerer Zeit werden die möglichen schädlichen metabolischen und entzündlichen Effekte von Lidocain sowohl auf das Spenderfett als auch auf die Empfängerzone diskutiert [17]. Lidocain in den üblichen Konzentrationen der Klein-Lösung von 0,05 bis $0,1 \%$ kann in vitro bei Zellkulturen den Glukose-Transport hemmen. Diesen Effekt beobachtet Moore [20] aber nur bei unmittelbarem Kontakt der Fettzellen zum Anästhetikum. Der gleiche Autor postuliert, dass diese potentiell ungünstigen Einflüsse auf die Zellviabilität nur während der Wirkungsdauer des Lokalanästhetikums (Lido- cain: $1-3 \mathrm{~h}$ ) zu beobachten ist und zudem reversibel ist. Keck [16] untersuchte menschliche Präadipozyten, welche zuvor während 30 min mit Bupivacain, Mepivacain, Ropivacain, Articain/Epinephrin, Lidocain und $\mathrm{NaCl}$ behandelt wurden. Die unmittelbaren Effekte von Mepivacain und Ropivacain waren moderat. Bei den mit Articain/Epinehphrin und Lidocain behandelten Zellen fanden sich jedoch vermehrt Schädigungen. Im Langzeitversuch beeinflusste nur Articain/Epihenphrin die Viabilität. Keck kommt zum Schluss, dass zwar die Zellen phänotypisch nicht geschädigt werden, dass hingegen alle getesteten Lokalanästhetika einen ungünstigen Einfluss auf die Differenzierung von Adipozyten haben. Die klinische Erfahrung zeigt, dass mit Lidocain eine genügende Anästhesie erreicht werden kann. Mit einer Vitalitätsrate von $80 \%$ (gegenüber $90 \%$ in der NaCl-Kontrolle) ist Lidocain daher immer noch das Lokalanästhetikum der Wahl. Um die Lidocainkonzentration in den Fettzellen zu reduzieren, empfiehlt Alexander [17] das Waschen des Fettkonzentrates mit Kochsalzlösung. Bei der WAL-Methode kommt im Vergleich zur klassischen Tumeszenzmethode relativ wenig Flüssigkeit zum Einsatz, wodurch der potentiell ungünstige Einfluss von Lidocain reduziert wird. Keck untersuchte in seiner Studie den Einfluss des pH-Wertes der Substanzen der Tumeszenzlösung auf die Zellviabilität der Adipozyten und kommt zum Schluss, dass die Viabilität bei der gepufferten Lösung bis zu $11 \%$ höher ist als bei der nichtgepufferten Lösung [16]. Die Zusammensetzung der Tumeszenzlösung ( $\bullet$ Tab. 2) bietet Gewähr, dass die Liposuction und die Aufbereitung des Fettes bezüglich der Zellviabilität zu jederzeit im optimalen pH-Milieu ablaufen. „Blut ist der Feind von Fett“ [21]: Blutbeimengungen im Transplantat können eine entzündliche Reaktion des Fettgewebes hervorrufen und die Makrophagenaktivität stimulieren. In der Folge werden Zellen resorbiert. Blut kann außerdem die Neovaskularisation hemmen und ist ein gutes Kulturmedium für das Wachstum von Bakterien. Aus diesen Gründen ist die Zugabe von Adrenalin in der Klein-Lösung unabdingbar. Adrenalin als Vasokonstriktor scheint keinen Einfluss auf die Viabilität der Fettzellen zu haben [20]. Jeder Adipozyt ist von Kapillaren umgeben. Diese Kapillaren sind extrem epinephrinempfindlich, dadurch bewirkt der Adrenalinzusatz in der Klein-Lösung eine ausgeprägte Vasokonstriktion und somit eine effiziente Blutstillung. Durch die Adrenalinbeigabe in der Spülflüssigkeit, die schonende Absaugtechnik mit dem Wasserstrahl und die Methode der Fettaufbereitung sind Blutbeimengungen bei der BE$\mathrm{AULI}^{\mathrm{TM}}$ - Methode äußerst minim. Bezüglich der Temperatur der Klein-Lösung finden sich in den meisten Arbeiten keine Angaben, es kann davon ausgegangen werden, dass die Temperaturen um die $20^{\circ}$ liegen. Fournier [12] appliziert die Lösung gekühlt, was möglicherweise die Blutungsneigung reduziert. Allerdings leidet der Patientenkomfort und die Fettzellen könnten durch die gekühlte Lösung geschädigt werden [15]. Aus diesen Gründen erwärmen wir die Klein-Lösung auf $37^{\circ}$.

\section{Liposuction}

Die Größe der Absaugöffnungen bestimmt die Größe der Fettgewebsstückchen. Die von uns verwendeten Kanülen weisen $0,9 \mathrm{~mm}$ große Öffnungen auf, damit lassen sich die Adipozyten atraumatisch und in genügend kleinen Partikeln gewinnen, ohne dass die Kanüle verstopft. Scharfe Kanülen bieten möglicherweise Vorteile in Bezug auf den Volumengewinn in der Empfängerzone. Eine Dissektion durch eine Kanüle mit scharf geformter Spitze scheint die Fettzellen mehr zu schonen als eine stumpfe Kanüle. Tulip ${ }^{\mathrm{R}}$ Medical bietet Kanülen mit speziell behandelten 
Oberflächen an („Superpolished Cannulas“), wodurch das Aspirat beim Passieren weniger beschädigt werden soll.

Histologische Untersuchungen zeigten, dass bei stärkerem Sog als -0,5 bar die Membranen der Zellen rupturieren und die Fettzellen vaporisieren. Ein maximaler Druck von -0,95 bar führt zu einer erhöhten Rate an destruierten Adipozyten. Shiffman [22] fand bei tieferen als $-700 \mathrm{mmHg}$ Drucken eine signifikante Erhöhung des Anteils an destruierten Zellen, bei Drucken von weniger als - $500 \mathrm{mmHg}$ hingegen eine histologisch gute Viabilität der Zellen. In Übereinstimmung mit diesen Befunden wird bei der BEAULI ${ }^{\mathrm{TM}}$-Methode der Druck bei der Aspiration auf -0,5 bis maximal $-0,55$ bar limitiert. Ein noch geringerer Unterdruck führt zu häufigem Verstopfen der Absaugkanüle und verringert die Effizienz der Fettgewinnung [4]. Neben diesen einstellbaren Grenzwerten sollte das Fett aber auch nicht abrupten Druckschwankungen ausgesetzt werden, zum Beispiel wenn die Liposuction unterbrochen wird. Starke Druckschwankungen werden durch die Konstruktion des Collectors ausgeglichen. Am Operateur liegt es, das Bypassloch am Kanülenhandstück zur Regulierung des Vakuums mit dem Daumen möglichst sanft abzudecken und freizugeben. Verschiedene Autoren weisen darauf hin, dass ein Luftkontakt des Aspirates möglichst zu vermeiden ist [5], weil dadurch die Qualität der Adipozyten und die Sterilität beeinträchtigt werden können.

\section{Fettaufbereitung}

Ziel der Aufbereitung ist, das Aspirat bezüglich Adipozyten, Wachstumsfaktoren und Stammzellen zu konzentrieren und von Blut, Serum und zerstörten Fettzellen zu separieren. Einige Autoren propagieren die Zentrifugation des Aspirates zur Separierung von Wasser, Blut und freien Lipiden [5, 21]. Übermäßiges Zentrifugieren kann aber die Adipozyten und die Fettstammzellen zerstören. Ein Zentrifugation erschwert überdies das Handling bei größeren Fettmengen bezüglich Sterilität, Zeitaufwand und des Vermeidens von Luftkontakt. Gemäß Angaben von anderen Autoren führt auch die Sedimentation des Transplantates zum gleichen Ziel wie die Zentrifugation, wird aber als schonenderes Vorgehen bezeichnet [5]. Ramon [6] zeigte im Tierversuch, dass die Einwachsrate von durch Sedimentation aufbereitetem Fett höher ist als von Fett, welches durch Zentrifugation gewonnen worden ist. Khater [7] kommt zum Schluss, dass eine Zentrifugation die Konzentration der Präadipozyten senkt und eine weniger ausgeprägte Zellproliferation bewirkt. Rose [8] verglich 3 Methoden der Zellseparierung, nämlich Zentrifugation mit Waschen, Zentrifugation ohne Waschen und alleinige Sedimentation. Die Ergebnisse zeigten, dass die durch Sedimentation gewonnenen Zellen die höchste Viabilität und den geringsten Volumenverlust an der Empfängerzone aufwiesen. Bei der BEAU$\mathrm{LI}^{\mathrm{TM}}$-Methode werden die Fettzellen durch die WasserstrahlTechnik und Funktionsweise des Collectors mit Filter- und Sedimentationseffekt soweit konzentriert, dass eine für die Zellen schädliche Zentrifugation nicht nötig ist. Eine Zentrifugation kann auch einen unerwünscht tiefen Restanteil an Flüssigkeit im Transplantat bewirken. Bei unserer Methode liegt dieser bei 30\% [4], dies erlaubt eine leichte Reinjektion und führt zu einer gleichmäßigen Verteilung im Empfängergewebe.

Unterschiedlich sind die Meinungen zum Waschen des Fettes: einige Chirurgen waschen das Aspirat mit $\mathrm{NaCl}$, Ringerlösung oder sterilem Wasser mit dem Ziel, das Überleben der Fettzellen dadurch zu verbessern. Gegen das Waschen sprechen der traumatisierende Effekt auf die empfindlichen Fettzellen und die Tatsache, dass durch das Waschen die Mikroumgebung der Zel- len (Kollagene, Gefäße, Proteine, Proteasen, Enzyme, Elektrolyte) gestört wird [23] und außerdem das Fibrin, welches die Adipozyten im Wundbett stabilisiert, herausgespült wird. Smith [24] untersuchte verschiedene Kombinationen der Fettaufbereitung mit nur Waschen, nur Zentrifugieren sowie Waschen und Zentrifugieren. Keine dieser Präparationstechniken zeigte Vorteile bezüglich Viabilität gegenüber nicht behandelten Fettzellen. Diese Erfahrungen und die Tatsache, dass bei der WAL die Fettpartikel bereits bei der Liposuction schonend „herausgespült" werden, bestätigen unsere Auffassung, dass ein Waschen und/oder Zentrifugieren keine Vorteile bringt.

\section{PRP und Stammzellen}

In einigen Studien wird postuliert, dass Platelet-Rich Plasma (PRP) die Einwachsrate eines Transplantates erhöhen soll [13]. Thrombozyten haben nicht nur die Eigenschaft, hämostatische Prozesse zu aktivieren, sondern sie initiieren auch die Entzündungskaskade, produzieren verschiedene Wachstumsfaktoren, Cytokine und spielen eine Rolle in der Wundheilung, in der Neovaskularisation sowie in der Induktion von mesenchymalen Stammzellen. PRP wird durch Zentrifugation aus dem Blut des Patienten gewonnen und mengenmäßig zu 0,1-1\% dem Transplantat beigemischt. Nakamura [25] konnte 2010 in einer Studie mit autologem Fetttransfer an 344 Ratten durch PRP eine signifikant höhere Rate an Gefäßneubildungen, Granulationsgewebe und intakten Adipozyten nachweisen. Salgarello [10] fand 2011 hingegen bei 42 Patientinnen mit Eigenfettbrustaufbau keinen Effekt von PRP im Vergleich mit der Kontrollgruppe. Die Zugabe von Bioaktivatoren zum Transplantat kann möglicherweise die Einwachsrate verbessern, das Auftreten von Ölzysten und Mikroverkalkungen minimieren sowie den Zellverlust reduzieren. Menschliche Adipozyten sind bis zu 0,1 mm groß, ihnen angelagert sind Präadipozyten bzw. Stammzellen, aus welchen sich neue Fettzellen entwickeln. $1 \mathrm{~g}$ menschliches Fett enthält zirka 550000 Stammzellen. Das Celution ${ }^{\mathrm{R}}$-System (Cytoris Therapeutics) soll in der Lage sein, Stamm- und Regenerativzellen von Fettgewebe zu isolieren. Ein enzymatischer Prozess befreit die Zellen von ihrer extrazellulären Matrix. Durch die anschließende Zentrifugation wird eine Konzentration der Stammzellen erreicht. Die aufwändige Prozedur ist im Versuchsstadium und wird nur von wenigen Autoren angewendet.

Yoshimura [26] beschreibt 2007 in einer Studie die Methode des zell-assistierten Lipotransfers (CAL) an 70 Patientinnen. Seine Überlegung beruht auf der Beobachtung, dass aspiriertes Fett im Vergleich zu intaktem Fett gefäßarm ist und eine tiefe Konzentration an ASCs (Adipose derived stromal cells/stem cells) aufweist. Aus der einen Hälfte des gewonnenen Fettes wird durch enzymatische Kollagenaseverdauungsreaktionen der extrazellulären Matrix, Zentrifugation und Elektrolyse die stromal vascular fraction (SVF), welche die ASCs enthält, isoliert. Diese Fraktion wird anschließend mit der anderen Hälfte des abgesaugten Fettes vermischt. Dieser Prozess ermöglicht es, aus einem ASCarmen Fettaspirat ein ASC-reiches Fett zu gewinnen. Tabit [27] untersuchte den Effekt von ASCs in der regenerativen Medizin und ihr Potenzial bezüglich Wundheilung und kommt zum Schluss, dass die Zugaben von aus der stromalen vaskularen Fraktion gewonnen ASCs die Einwachsrate und das Überleben von Fetttransplantaten fördern könnte. Brayfield beschreibt in seiner Studie von 2010 die Möglichkeit, aus dem menschlichen Fettgewebe ASC zu isolieren, die sich in reine Adipozyten entwickeln können [28]. Die Autoren weisen insbesondere auf das angiogenetische Potenzial der ASCs und heben so die Bedeutung 
dieser Zellen auf dem Gebiet der Transplantation hervor. 2012 kommt Philips in seiner Arbeit zum Schluss, dass die ASCs, obschon Standards in der Gewinnung und Verarbeitung noch fehlen, in Zukunft eine bedeutende Rolle in der Verbesserung der Einheilungsraten bei autologen Fetttransplantationen spielen werden [29].

Anhand der Oberflächenzellmarker lassen sich multiple Zellen aus der SVF typisieren: neben der bereits erwähnten ASC auch endotheliale Progenitorzellen und Perizyten. Largo [5] untersuchte die SVF bezüglich Zellqualität und -komposition von Proben aus verschiedenen Entnahmeregionen und konnte dabei keine Unterschiede feststellen. Eine negative Korrelation konnte er jedoch zwischen dem Alter des Patienten und der relativen Progenitorzellzahl nachweisen. Die Autoren schlossen daraus, dass dies auf ein reduziertes Vaskularisierungspotenzial des Fetttransplantates bei älteren Patienten und somit eine schlechtere Einheilungsrate deuten könnte. In ihrer klinischen Studie postuliert der Autor, dass die endotheliale Progenitorpopulation der frisch isolierten SVF oder das mittels Zentrifugation gewonnene Lipoaspirat die Neovaskularisation der transplantierten Fettzellen unterstützen. Ueberreiter [4] postuliert, dass nicht eine Stammzellanreicherung die Einheilung der Adipozyten fördert, sondern dass die Nähe zu einem Blutgefäß entscheidend ist. Außerdem benötigt man zur Isolierung von genügend Stammzellen zusätzlich etwa $500 \mathrm{ml}$ Fett, was aber bei eher schlanken Patientinnen schwierig zu gewinnen ist. Die Prozedur dauert 2-3 h, dazu fallen erhebliche Mehrkosten an.

Kaminski [23] untersuchte die Wirkung von Albumin auf das Transplantat: das Infiltrieren der klassischen Tumeszenzlösung kann aufgrund der massiven Verdünnung der wasserlöslichen Proteine einen osmotischen Schock der Fettzellen zur Folge haben und somit das Überleben der Zellen ungünstig beeinflussen. Der Autor empfiehlt zum Ausgleich des Kolloiddruckes die Zugabe von $10 \%$ humanem Serum Albumin in das entnommene Transplantat. In den meisten Studien über weitere Additive wie Insulin, Heparin, Kalzium, Schilddrüsenhormone oder Vitamin E konnte keine nennenswerte Beeinflussung des Volumenzuwachses durch solche Substanzen nachgewiesen werden [13].

\section{Brava}

Ein Problem bei der Brustvergrößerung kann sein, dass die Fettzellen gleichzeitig als Expander und auch als natürlicher Gewebefüller dienen. Bei kleinen oder straffen Brüsten bzw. wenn relativ große Volumen verpflanzt werden, kann die Spannung im Gewebe die Durchblutung des Empfängerbettes und der Haut beeinträchtigen, lokale Ischämien verursachen und dadurch die Einwachsrate reduzieren. Khouri empfiehlt daher, das Gewebe vorgängig mit dem nichtchirurgischen BRAVA ${ }^{\mathrm{R}}$-System (Brava, LLC, USA) zu expandieren [11]. Allerdings kann diese Technik auch zu Schmerzen, Ödemen und Gewebeläsionen führen [13], zudem ist die Compliance als eher niedrig einzustufen. Ohnehin sollte das Empfängergewebe nicht überfüllt werden oder der Eingriff in mehreren Etappen geplant werden, wodurch sich die Indikation zum Einsatz der Brava-Methode relativiert.

\section{Kryokonservation von Fett}

Um die Anzahl der Liposuctionen bei 2 oder mehr Transfer-Operationen zu reduzieren, wäre die Kryokonservation von Fett wünschenswert. Raskin [30] findet in seiner Literaturübersicht zur Kryokonservation von Fett Unterschiede in den Empfehlungen bezüglich Temperatur ( +4 bis $-70^{\circ}$, langsames oder schnelles Einfrieren und Auftauen) und Dauer der Konservation (bis zu
3 Jahre). Die Studien untersuchen das konservierte Fett bezüglich Veränderungen in Volumen, Gewicht, histologischen Parametern, Viabilität, Stammzellkonzentration, bakterieller Kontamination und klinischen Verläufen. Die Ergebnisse reichen von keine signifikante Unterschiede bis zu 50\% Volumenverlust, 20\% Gewichtsverlust und vermehrte Fibrose- und Cystenbildung sowie schlechtere histologische Parameter. Die Angaben in der Literatur lassen den Schluss zu, dass eine Kryokonservation des Fettes aufgrund der Komplikationsmöglichkeiten und der reduzierten Resorptionsraten außer Betracht fällt. Matsumoto [15] empfiehlt, dass aspiriertes Fett sobald wie möglich wieder infiltriert werden sollte, bereits nach einer Konservierung bei Raumtemperatur über $4 \mathrm{~h}$ fand er eine Zunahme des Ölvolumens.

\section{Reinjektion}

Die Infiltration ist ein entscheidender Faktor für eine erfolgreiche Fettzell-Transplantation. Die Fettzellen können noch von so guter Qualität sein - wenn das Transplantat bei der abschließenden Infiltration unregelmäßig, in Klumpen, in falsche Schichten oder mit zu hohem Druck verteilt wird, ist der Misserfolg programmiert. Zur Infiltration verwenden die meisten Autoren $10 \mathrm{ml}$ Spritzen. Der Durchmesser der Infiltrationskanüle sollte mindestens $18 \mathrm{G}$ betragen. Bei kleinerem Nadeldurchmesser kann die metabolische Aktivität der Fragmente beeinträchtigt werden, weil der Druck bei der Injektion als Funktion des abnehmenden Kanülendurchmessers zunimmt. Die meisten Autoren geben stumpfen Kanülen den Vorzug, scharfe Kanülenspitzen können unerwünschte Blutungen verursachen. Bei unserer Serie arbeiteten wir mit stumpfen Kanülen von $150 \mathrm{~mm}$ Länge, 2,7 mm Außendurchmeßer (12G) und 2 seitlich um $180^{\circ}$ versetzte Öffnungen mit einem Durchmesser von 1,2 mm. Damit ist gewährleistet, dass die Fettpartikel gleichmäßig, ohne zu großen Stempeldruck und mit minimaler Traumatisierung des Empfängergewebes verteilt werden, ohne dass die Kanüle verstopft.

Die Fettpartikelinjektionen erfolgen in die subkutanen und subglandulären Fettschichten sowie im Bereich der Pectoralismuskulatur. Alle Autoren vermeiden eine Infiltration in das Brustdrüsengewebe, einzig Illouz [1] infiltriert intraglandulär. Herold [9] fand in seiner MRT-volumetrisch gemessenen Reihe 6 Monate nach der Fettzellverpflanzung einen Volumenerhalt von $81 \%$ im periglandulären Fettgewebe und einen geringeren Volumenerhalt von 64\% im Muskel. Offen bleibt die Frage, ob Muskulatur als Empfängergewebe von Fett bezüglich Haltbarkeit, Form, Festigkeit, Funktion und Biokompatibilität die gleichen Eigenschaften wie Fettgewebe als Empfängerzone aufweist. Das Überleben des Transplantates ist von der Vaskularisation und von der Diffusion mit einer maximalen Distanz von $100 \mu \mathrm{m}$ abhängig. Diese Tatsache könnte bei einem Transfer in die Muskulatur als Vorteil betrachtet werden, weil die Muskulatur als Empfängermatrix besser durchblutet ist als Fett. Die Pectoralismuskulatur bietet sich als zusätzliches Zielvolumen an, um gerade bei kleineren Brüsten das sicher injizierbare Gesamtvolumen zu erhöhen. Selten finden sich Angaben bezüglich Lokalisation und Anzahl der Hautinzisionen für die Reinjektion. Ueberreiter [4] empfiehlt nur eine Inzision lateral im Bereich der vorderen Axillarlinie, zirka 1-2 cm von der Brust entfernt. Um auch den oberen inneren Quadranten zu erreichen und um eine gleichmäßigeres Filling zu erzielen, setzen wir eine zusätzliche Inzision parasternal $(\bullet$ Abb. 3).

Es ist nicht möglich, den endgültigen Volumengewinn durch eine Überfüllung verbessern zu wollen. Der dabei entstehende zusätzliche Druck kann die Durchblutung beeinträchtigen, Fett- 
zellen zerstören und somit das Risiko der Entstehung von Zysten und Kalkablagerungen erhöhen (Overfilling $\rightarrow$ Necrosis und Overgrafting $\rightarrow$ Loosing Fat). Illouz [1] empfiehlt tendenziell ein Vorgehen in mehreren Sitzungen mit jeweils geringeren Transplantat-Volumina, dadurch kann die Einheilungsrate verbessert werden, insgesamt ein größerer Volumenzuwachs erreicht werden und gleichzeitig das Risiko von Komplikationen reduziert werden. Wird ein größerer Volumenzuwachs gewünscht, kann den Patientinnen ein weiterer Eingriff angeboten werden. Voraussetzung ist, dass genügend Spenderfett zur Verfügung steht. Der zeitliche Abstand zu einem Zweiteingriff sollte 4-6 Monate betragen. 2 oder mehr Eingriffe sind auch zu planen, wenn bei sehr kleinen Brüsten, Status nach Ablatio oder bei Status nach Radiotherapie mit sehr rigidem, vernarbtem Gewebe nur eine dünne Empfänger-Fettschicht vorhanden ist. Da die Fettschicht mit jedem Eingriff an Volumen zunimmt, kann mit jedem weiteren Transfer auch mehr Volumen eingebracht werden.

\section{Resultate}

Die Erfolgskontrolle basiert auf den subjektiven Aussagen der Patientinnen wie größere, vollere oder prallere Brüste sowie auf dem vorher/nachher Vergleich von standardisiert aufgenommenen Fotos seitlich. Um bei den seitlichen Aufnahmen reproduzierbare Vergleiche zu erhalten, sollte die Aufnahme exakt in der verlängerten Achse der beiden Mamillen erfolgen. Wichtig ist auch die Bestimmung des Körpergewichts vor der Operation und bei der Nachkontrolle. Gewichtsschwankungen haben auch Änderungen im Brustvolumen zur Folge. Ueberreiter [4] empfiehlt zur Kontrolle vorher/nachher die Messung des Abstandes zwischen Jugulum und Mamille sowie den maximalen Einzelbrustumfang, gemessen vom medialen Ansatz über die Mamille bis zum lateralen Ansatz auf der Höhe der vorderen Axillarlinie. Der Autor weist darauf hin, dass diese Masse zwar kein exaktes Messkriterium darstellen, in der Summe jedoch einen guten Anhalt für das Einheilungsverhalten des Fettes darstellen. Volumetrische Auswertungen mittels MRT scheinen in Bezug auf den gleichzeitigen Nachweis von strukturellen Veränderungen und Genauigkeit der Resultate die überlegene Methode zu sein. Die Messungen zeigten, dass sich der Volumenrückgang in den ersten 4 Wochen abspielte, danach konnten nur noch minime Änderungen registriert werden.

Ueberreiter [4] führte mit der gleichen Technik wie bei uns bei 85 Patientinnen einen Eigenfett-Brustaufbau durch. Ein MRT wurde präoperativ und in 72 Fällen mindestens 6 Monate postoperativ durchgeführt. Das Infiltrat setzt sich aus 68\% (60-72\%) Fett und 32\% (18-40\%) Wasser zusammen. Die Einheilungsrate betrug 76\% (65-87\%). Daraus ergibt sich eine Nettovolumenzunahme der Brust in der Höhe von 51,7\% des eingespritzten Volumens. Largo [5] bestimmt in seiner laufenden Studie, allerdings ohne Angaben über Fallzahlen, die Brustvolumina prä- und postoperativ mit einem 3-dimensionalen Laserscanner. Dabei variieren die Fett-Überlebensraten selbst bei gleichem Operateur und identischem Operationsverfahren zwischen 25\% und $90 \%$. Ein hoher Graftverlust sei primär auf eine hohe Reabsorption und/oder auf Nekrosen zurückzuführen. Der Autor empfiehlt, gegebenenfalls wiederholte Eingriffe durchzuführen.

\section{Komplikationen}

Fetttransfer-Operationen können mit Komplikationen behaftet sein. Übliche, vorübergehende Nebenwirkungen sind Schwellungen, Rötungen, Volumenverlust nach einigen Wochen, Pruritus, Unregelmäßigkeiten der Oberfläche oder Allergien auf Anti- biotika. Illouz [1] berichtet in 4,1\% der Fälle über Asymmetrien der Brüste in den Langzeitkontrollen. Einheilungsraten zwischen 0 und $70 \%$ können die Folge von Blutbeimengungen, Infektionen, unkorrekter Entnahmetechnik, zu hohem Vakuum, fibröser Spenderzonen oder der Verwendung von zerstörten Adipozyten sein. Die Injektion von zu großen Transplantat-Portionen kann zur Bildung von Zysten führen, da nicht alle abgestorbenen Adipozyten von den Makrophagen abtransportiert werden. Ölzysten entstehen aus einer Anhäufung von nekrotischen Fettzellen, die Rückbildung kann über Jahre dauern [21]. Verkalkungen können wie bei jeder Brustoperation auftreten. Nach Largo [5] wird das Risiko von Kalzifikationen und Ölzysten in der Literatur bis zu 50\% angegeben, Ueberreiter beschreibt eine Häufigkeit von 4,5\% [4]. Cotrufo [31] weist darauf hin, dass eine bildgebende prä- und postoperative Untersuchung bei jeder Filling-OP durchgeführt werden muss. Der Autor vermutet, dass bei größeren transplantierten Fettmengen das Risiko der Entstehung von Verkalkungen und Nekrosen zunimmt. In seinen eigenen 42 Fällen betrug die durchschnittliche transferierte Fettmenge daher nur $197 \mathrm{ml}$. In der Literaturübersicht von 60 Publikationen mit 4601 Patientinnen von Claro [32] 2012 fanden sich in 3,9\% Komplikationen, die meisten waren Indurationen und/ oder palpable Verhärtungen. Infektionen sind selten, werden aber in Einzelfällen beschrieben. Die präoperativen Kontrollen des weißen Blutbildes, des Blutzuckers, Hepatitis B und C-Screening sowie eine HIV-Test sollen Patientinnen mit erhöhtem Infektionsrisiko ausschließen. Aktive Infektherde (Tonsillen, Abszesse) sind absolute Kontraindikationen für einen Fetttransfer. Infekte können durch eine strikte aseptische Operationstechnik und durch den Einsatz eines sterilen, geschlossenen Absaugund Aufbereitungssystems ohne Luftkontakt vermieden werden. Die Liposuction sollte unter minimalem Blutverlust erfolgen und das Infiltrat sollte blutfrei sein. Die Operationszeiten müssen sich in einem vernünftigen Rahmen bewegen und inklusive Absaugung nicht länger als $2-2 \frac{1}{2} \mathrm{~h}$ dauern. Die wiederholte Desinfektion der Inzisionsstellen bei jedem erneuten Einführen der Kanüle, das strikte Vermeiden des Berührens der Kanüle und eine Antibiotikaprophylaxe bieten zusätzliche Sicherheit.

Hyakusoku [33] beschreibt Fälle von Komplikationen nach Brustaufbau, wobei alle Eingriffe und von nicht ausgebildeten Ärzten durchgeführt worden sind. Bei 12 Patientinnen fanden sich in 3 Fällen palpable Indurationen, in 3 Fällen druckdolente Verhärtungen und in je einem Fall eine Infektion, eine Sekretion und eine Lymphadenopathie. 4 Patientinnen hatten Anomalitäten beim Screening nach der Operation. Das Auftreten eines Pneumothorax bei Fetttransplantationen in die Brust ist beschrieben. In der Literatur als Einzelfälle beschrieben werden Fettembolien, Strokes und lipoide Meningitiden, allerdings traten diese Komplikationen ausschließlich nach Fettinjektionen im Gesichtsbereich auf [5].

\section{Bildgebende Brustvorsorgeuntersuchungen und Tumorinduktion}

Der Brustaufbau mit Eigenfett wird bezüglich Einschränkungen der Brustkrebsdiagnostik und der möglichen Tumorindikation kontrovers beurteilt. Hauptkritikpunkt ist die Möglichkeit der Entstehung von Mikroverkalkungen, Zysten oder Narbensträngen, welche die Brustvorsorgeuntersuchungen konkomittieren können. Mikroverkalkungen können aber bei jeder anderen Brustoperation auftreten, zum Beispiel nach Vergrößerungen mit Implantaten, offener und geschlossener Kapsulotomie, Brustreduktionen, Biopsien, Bestrahlungstherapien und Brustli- 
posuktionen. In einer vergleichenden Studie von Mammografien vor und nach einem Brustfilling konnte Veber 2011 keine signifikanten Unterschiede bezüglich Verkalkungen und zystischen Läsionen nachweisen [34]. Der Autor schließt daraus, dass die radiologische Verlaufskontrolle nach Brustaugementationen mit Eigenfett unproblematisch ist und keinen Hinderungsgrund für diese Methode darstellt. Kalzifikationen treten laut Coleman beim Lipofilling nicht mehr auf als bei anderen Brusteingriffen [35]. Coon [36] verglich 2011 die postoperativen Mammografien nach Brustaufbau und nach Reduktionsmammoplastiken. Dabei fand er in der Gruppe der Reduktions-Operationen in den Kontroll-Mammografien mehr Vernarbungen, welche eine Biopsie erforderten als in der Vergleichsgruppe der Patientinnen mit Eigenfett-Brustaufbau. Bezüglich Ölzysten und gutartigen Verkalkungen fanden sich keine signifikanten Unterschiede. Rubin fand 2012 bei 27 Patientinnen nach Eigenfett-Brustaugmentation weniger radiologische Abnormitäten gegenüber einer Vergleichsgruppe mit 23 Patientinnen nach Reduktionsplastik [37]. In der Literaturzusammenstellung von Claro [32] 2012 fanden sich bei 2560 operierten Frauen in 13,0\% radiografische Anomalien, in mehr als der Hälfte in Form von Zysten. Mit den bildgebenden Verfahren wie Mammografie, Sonografie und MRI lassen sich Kalzifikationen in Zusammenhang maligner Prozesse von benignen Verkalkungen, verursacht unter anderem durch Brustoperationen oder im speziellen auch Filling-Eingriffen, unterscheiden [5]. Bisher gibt es keinen beschriebenen Fall einer verzögerten Mammakarzinom-Diagnostik wegen Verkalkungen nach einem Eigenfetttransfer [38].

Seit der ersten Publikation von autologen Fettgewebetransplantationen in die Brust vor mehr als 100 Jahren sind auch nach langen Verlaufskontrollen keine wesentlichen negativen Folgen, insbesondere bezüglich einer möglichen Karzinomentwicklungen, beschrieben worden [4]. Rigotti [39] verglich 2010 die Inzidenz von lokalen und regionalen Rezidiven von Mammakarzinomen bei 137 Patientinnen, welche sich nach einer modifizierten radikalen Mastektomie einem Eigenfett-Brustaufbau unterzogen. Nach einer mittleren Beobachtungszeit von 7,6 Jahren fand sich eine vergleichbare Inzidenz von Lokalrezidiven. In der Zusammenstellung von Claro [32] betrug die Rate eines Lokalrezidivs bei Brustaufbau mit Eigenfett nach Ablatio bei 616 Patientinnen 2,3\%. Der Autor postuliert, dass die onkologische Sicherheit unklar bleibt. Illouz [1] kontrollierte 230 Patientinnen nach einem Eigenfetttransfer in die Brust während durchschnittlich 11,3 Jahren. Die jährlich durchgeführten Mammografien zeigten keine erhöhte Inzidenz von Brustläsionen, inklusive Verkalkungen, Zysten oder Karzinomen. Fraser [40] fand 2011 bei der Durchsicht von mehreren klinischen Studien mit autologem Fetttransfer bei über 2000 Patientinnen keine erhöhte Inzidenz von Karzinomen.

\section{Akzeptanz der Fachgesellschaften}

Autologer Fetttransfer für Brustaufbau und -rekonstruktion wurde Ende der 80-Jahre des letzten Jahrhunderts in den USA sehr kontrovers diskutiert. Schlechte Einheilungsraten, Verkalkungen, Fettnekrosen und unklare Mammografiebefunde brachten die Methode in Misskredit. Fortschritte in der Fettzellgewinnung und der Transfertechnik, digitale Mammografie und die Möglichkeit der Anreicherung von Stammzellen bewirkten ein Umdenken. Noch 1987 lehnte die American Society of Plastic Surgeons die autologe Fetttransplantation in die Brust ab, weil die Brustvorsorgeuntersuchungen kompromittiert würden [4]. 1992 äußerte sich die ASPS in einem Positionspapier zum Eigen- fettbrustaufbau. Dabei verurteilt sie die Methode weiterhin, weil dadurch die Früherkennung erschwert werde oder aber falsch positive Resultate im Screening die Folge sein können. Außerdem könnten Vernarbungen und Verkalkungen entstehen, welche chirurgische Biopsien oder gar Mastektomien erfordern. 2009 schließlich kommt die ASPS in einem Positionspapier neu zum Schluss, dass die Methode eine mögliche Therapieform darstellt, von der Technik und der Erfahrung des Chirurgen abhängt, dass die Patientinnen entsprechend aufgeklärt werden müssen und dass Patientinnen mit erhöhtem Mammakarzinomrisiko, positivem BRCA-1, BRCA-2, persönlicher oder familiärer Anamnese mit Brustkrebs mit Vorsicht auszuwählen sind. In Relation zu diesen Statements sind aber auch die Risiken und Folgen bei der Verwendung von Implantaten zu sehen. Kapselkontrakturen kommen in bis zu 70\% der Fälle vor, die Kapselfibrose um das Implantat kann ebenso Verkalkungen verursachen.

\section{Schlussfolgerung}

Die Verpflanzung von autologen, lebenden Adipozyten und Präadipozyten zum Zweck einer Konturverbesserung oder eines Volumengewinnes hat in den letzten Jahren an Stellenwert gewonnen und ist zu einem Routineeingriff geworden. Die Sicherheit, Effizienz und das Resultat eines Eigenfetttransfers in die weibliche Brust ist abhängig von der Technik. In der Literatur werden anhand von Fallserien, Case Reports und Expertenmeinungen unterschiedliche Methoden der Fettgewinnung, der Präparation, der Speicherung und der Injektion beschrieben. Systematische Kontrollen oder qualitativ hochstehende randomisierte Studien mit hohen Fallzahlen und Untersuchungen über den Langzeitverlauf fehlen. Notwendig sind auch die Erarbeitung von Standards um die Ergebnisse reproduzieren zu können sowie qualitativ-quantitative Analysen des Augmentationsvolumens. Die Verwendung von Additiven zur Bioaktivation von Transplantat und Empfängergewebe um die Resorptionsrate zu erhöhen ist von substanziellem Interesse und muss weiter untersucht werden. Eine weitere Reduktion des Fettverlustes und der Inzidenz der Entstehung von Zysten und Mikroverkalkungen ist anzustreben. Alle potentiellen Methoden zur weiteren Optimierung der Resorptionsrate dürfen die Sicherheit des Fetttransfers großer Volumina nicht beeinträchtigen und müssen mit vernünftigem Aufwand praktisch durchführbar sein.

Körperfremde Filler wie Silicone, Kollagen und Hydroxyapatit, aber auch autologe Materialien wie Hautlappen, lokale oder freie Lappen haben beträchtliche Nachteile und können zu enttäuschenden Resultaten führen. Manche Patientinnen haben Angst vor den möglichen Problemen der Implantate wie Fremdkörperreaktionen, Narben, Asymmetrien, Schmerzen, Volumenverlusten, Kapselkontrakturen, Unebenheiten oder unnatürlichem Aussehen. Die autologe Fettzell-Transplantation in die weibliche Brust ist eine gute und sichere Methode, eine sanfte, harmonisch wirkende Brustvolumenvergrößerung sowie eine nachhaltige und natürlich wirkende Konturverbesserung zu erzielen. Ausgewählte und aufgeklärte Patientinnen im mittleren Lebensalter, welche eine Abneigung gegenüber Fremdkörpern haben, vollere Brüste oder einen Ausgleich bei Asymmetrien wünschen, sind mit den Resultaten dieser an Bedeutung laufend zunehmenden Methode sehr zufrieden. 


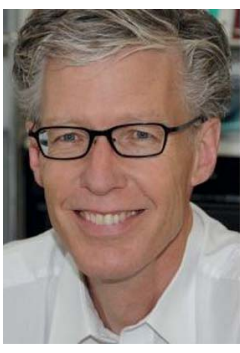

Daniel Münch,

31. Januar 1955 geboren in Zürich, 1974-1980 Studium der Medizin Universität Bern, Staatsexamen 1980, 1980-1990 Bezirksspital Niederbipp (Chirurgie); Universitätsspital Bern (Viszerale Chirurgie, Thorax- Herz- und Gefäßchirurgie, Neurochirurgie, Kinderchirurgie, Urologie); Kantonsspital Aarau (Chirurgie). 1983 Dissertation, 1990 Facharzt für Chirurgie, 1993-1995 Stage Prof. Fournier, seit 1990 als selbstständiger Chirurg mit Praxis für Liposuctionen und ambulante Chirurgie in Wiedlisbach/Bern und Belegarzttätigkeit Klinik Villa im Park, Rothrist. Schwerpunkte seit 1993: Liposuctionen. Mitglied der Schweizerischen Gesellschaft für Chirurgie.

\section{Interessenkonflikt: Nein}

\section{Literatur}

1 Illouz YG, Sterodimas A. Autologous Fat Transplantation to the Breast: A Personal Technique with 25 Years of Experience. Aesth Plast Surg 2009; 33: 706-715

2 Coleman SR, Saboeiro AP. Fat Grafting to the Breast Revisited: Safety and Efficacy. Plast Reconstr Surg 2007; 119: 775-785

3 Coleman SR. Structural Fat Grafting. Aesthet Surg J 1998; 18: 386-388

4 Ueberreiter $K$, von Finckenstein JG, Cromme F et al. BEAULI ${ }^{\mathrm{TM}}$ - eine neue Methode zur einfachen und zuverlässigen Fettzell-Transplantation. Handchir Mikrochir Plast Chir 2010; 42: 379-385

5 Largo RD, Häcki J, Güven $S$ et al. Autologe Fetttransplantation in die weibliche Brust. Schweiz Med Forum 2011; 11: 489-494

6 Ramon Y, Shoshani O, Peled IJ et al. Enhancing the Take of Injected Adipose Tissue by a Simple Method for Concentrating Fat Cells. Plast Reconstr Surg 2005; 115: 197-201

7 Khater R, Atanassova P, Anastassov Y et al. Clinical and Experimental Study of Autologous Fat Grafting after Processing by Centrifugation and Serum Lavage. Aesthet Plast Surg 2009; 33: 37-43

8 Rose JG Jr, Lucarelli MJ, Lemke BN et al. Histologic Comparison of Autologous Fat Processing Methods. Ophthal Plast Reconstr Surg 2006; 22: $195-200$

9 Herold C, Ueberreiter K, Cromme $F$ et al. Ist eine intramuskuläre Injektion bei autologer Fetttransplantation zur Mamma sinnvoll? Eine MRT-volumetrische Studie. Handchir Mikrochir Plast Chir. doi: 10.1055/s-0030-1269931

10 Salgarello M, Visonti G, Rusciani A. Breast Fat Grafting with PlateletRich Plasma: A Comparative Clinical Study and Current State of the Art. Plast Reconstr Surg 2011; 127: 2176-2185

11 Khouri R, Del Vecchio D. Breast Reconstruction and Augmentation using Pre-Expansion and Autologous Fat Transplantation. Clin Plast Surg 2009; 36: 269-280

12 Fournier PF. Fat grafting: My Technique. Dermatol Surg 2000; 26: $1117-1128$

13 Alexander RW. Use of Platelet-Rich-Plasma to Enhance Effectiveness of Autologous Fat Grafting. In: Shiffman MA. Autologous Fat Transfer. Berlin Heidelberg: Springer; 2010; 87-112

14 Shiffman MA (eds.). Commentary. In: Shiffman MA. Autologous Fat Transfer. Berlin Heidelberg: Springer; 2010; 463-465

15 Matsumoto $D$, Shigeura $T$, Sato $K$ et al. Influence of Preservation at Various Temperatures on Liposuction Aspirates. Plast Reconstr Surg 2007; 120: 1510-1517

16 Keck M, Janke J, Ueberreiter K. Viability of Praeadipocytes In Vitro: The Influence of Local Aenesthetics and pH. Dermatol Surg 2009; 35 : $1251-1257$
17 Alexander RW. Autologous Fat Grafting: A Study of Residual Intracellular Adipocyte Lidocaine. In: Shiffman MA. Autologous Fat Transfer. Berlin Heidelberg: Springer; 2010; 445-450

18 Shiffman MA. Fat Transfer Principles. In: Shiffman MA. Body Contouring. Berlin Heidelberg: Springer; 2010; 577-585

19 Rohrich RI, Sorokin ES, Brown SA. In Search of Improved Fat Transfer Viability: A Quantitative Analysis of the Role of Centrifugations and Harvest Site. Plast Reconstr Surg 2004; 114: 391-395

20 Moore JH Jr, Kolaczynski JW, Morales LM et al. Viability of Fat Obtained by Syringe Suction Lipectomy: Effects of Local Anesthesia with Lidocaine. Aesth Plast Surg 1995; 19: 335-339

21 Shiffman MA. Principles of Autologous Fat Transplantation. In: Shiffman MA. Autologous Fat Transfer. Berlin Heidelberg: Springer; 2010; $11-17$

22 Shiffman MA, Mirrafati S. Fat Tansfer Techniques: The Effect of Harvest and Transfer Methods on Adipocyte Viability and Review of the Literature. Dermatol Surg 2001; 27: 819-826

23 Kaminski MV, Lopez de Vaughan RM. Fat Autograft Retention with Albumin. In: Shiffman MA. Autologous Fat Transfer. Berlin Heidelberg: Springer; 2010; 123-133

24 Smith P, Adams WP Jr, Lipschitz AH et al. Autologous Human Fat Grafting: Effect of Harvesting and Preparation Techniques on Adipocyte Graft Survival. Plast Reconstr Surg 2006; 117: 1836-1844

25 Nakamura S, Ishihara M, Takikawa M et al. Platelet-Rich Plasma (PRP) Promotes Survival of Fat-Grafts in Rats. Annals of Plast Surg 2010; 65: 101-106

26 Yoshimura K, Sato K, Aoi N et al. Cell-Assisted Lipotransfer for Cosmetic Breast Augmentation: Supportive Use of Adipose-Derived Stem/ Stromal Cells. Aesth Plast Surg 2008; 32: 48-55

27 Tabit CJ, Slack GC, Fan K et al. Fat Grafting Versus Adipose-Derived Stem Cell Therapy: Distinguishing Indications, Techniques and Outcomes. Aesth Plast Surg doi:10.1007/s-00266-011-9835-4

28 Brayfield C, Marra K, Rubin JP. Adipose Stem Cell for Soft Tissue Regeneration. Handchir Mikrochir Plast Chir 2010; 42: 124-128

29 Philips BJ, Marra KG, Rubin JP. Adipose Stem Cell-Based Soft Tissue Regeneration. Expert Opinion on Biological Therapy 2012; 12: 155-163

30 Raskin BI. Cryopreserved Fat. In: Shiffman MA. Autologous Fat Transfer. Berlin Heidelberg: Springer; 2010; 305-311

31 Cotrufo S, Mandal A, Mithoff EM. Letter/Comment, Plastic and Reconstr Surgery doi:10.1097/01.prs.0000294957.94383.84

32 Claro F Jr, Figueiredo JC, Zampar AG et al. Applicability and Safety of Autologous Fat for Reconstruction of the Breast. Brit J Surg 2012; 99: 768-780

33 Hyakusoku H, Ogawa R, Ono S et al. Complications after Autologous Fat Injection to the Breast. Plast Reconstr Surg 2009; 123: 360-370

34 Veber $M$, Tourasse $M$, Tousson $G$ et al. Radiographic Findings after Breast Augmentation by Autologous Fat Transfer. Plast Reconstr Surg 2011; 127: 1289-1299

35 Coleman SR. Augmentation of the Breast with Structural Fat. In: Coleman SR. Fat Injection. From Filling to Regeneration. St. Louis: Quality Medical Publishing Inc; 2009; 503-544

36 Coon D, Zuley M, Toy J et al. Mammographic Changes after Stem Cell Supplemented Fat Transfer to the Breast Compared with Changes after Breast Reduction: A Blinded Study. Plast Reconstr Surg 2011; 128: 54

37 Rubin JP, Coon D, Zuley $M$ et al. Mammographic Changes after Fat Transfer to the Breast Compared with Changes after Breast Reduction: A Blinded Study. Plast Reconstr Surg 2012; 129: 1029-1038

38 Shiffman MA. Medical Legal Aspects of Autologous Fat Transplantation. In: Shiffman MA. Autologous Fat Transfer. Berlin Heidelberg: Springer; 2010; 459-462

39 Rigotti G, Marchi A, Stringhini P et al. Determing the Oncological Risk of Autologous Lipoaspirate Grafting for Post-Mastectomy Breast Reconstruction. Aesth Plast Surg 2010; 34: 475-480

40 Fraser JK, Hedrick M, Cohen S. Oncologic Risk of Autologous Fat Grafting to the Breast. Aesth Surg J 2011; 31: 68-75 